\title{
Novel hydroxamic acids incorporating 1-((1H-1,2,3-Triazol-4-yl)methyl)-3-hydroxyimino-indolin-2-ones: synthesis, biological evaluation, and SAR analysis
}

\author{
DO THI MAI DUNG ${ }^{\mathrm{a}}$, PHAM-THE HAI ${ }^{\mathrm{a}}$, DUONG TIEN ANH ${ }^{\mathrm{a}}$, LE-THI-THU HUONG ${ }^{\mathrm{b}}$, \\ NGUYEN THI KIM YEN ${ }^{c}$, BYUNG WOO HAN ${ }^{c}$, EUN JAE PARK ${ }^{\mathrm{d}}$, YEO JIN CHOI ${ }^{\mathrm{d}}$, \\ JONG SOON KANG ${ }^{\mathrm{e}}$, VAN-THI-MY HUE ${ }^{\mathrm{a}, *}$, SANG-BAE HAN $^{\mathrm{d}, *}$ and NGUYEN-HAI NAM ${ }^{\mathrm{a}, *} \mathbb{D}$ \\ ${ }^{a}$ Hanoi University of Pharmacy, 13-15 Le Thanh Tong, HoanKiem, Hanoi, Vietnam \\ ${ }^{\mathrm{b}}$ School of Medicine and Pharmacy, Vietnam National University, 144 XuanThuy, Hanoi, Vietnam \\ ${ }^{\mathrm{c}}$ Research Institute of Pharmaceutical Sciences, College of Pharmacy, Seoul National University, Seoul 151-742, \\ Korea \\ ${ }^{\mathrm{d}}$ College of Pharmacy, Chungbuk National University, Cheongju, Chungbuk 28160, Korea \\ ${ }^{\mathrm{e}}$ Bio-Evaluation Center, Korea Research Institute of Bioscience and Biotechnology, Cheongju, Chungbuk, Korea \\ E-mail: shan@chungbuk.ac.kr; namnh@hup.edu.vn
}

MS received 5 March 2018; revised 22 April 2018; accepted 29 April 2018; published online 1 June 2018

\begin{abstract}
A series of seventeen novel hydroxamic acids incorporating 1-((1H-1,2,3-triazol-4-yl)methyl)3-hydroxyimino-indolin-2-ones was designed and synthesized. Biological evaluation showed that these hydroxamic acids potently inhibited a class-I isoform of HDACs (HDAC2) with IC $_{50}$ values in low micromolar range. Several compounds also exhibited good cytotoxicity. Two compounds, $\mathbf{5 e}$ and $\mathbf{5 f}$, emerged as the most potent HDAC2 inhibitors with cytotoxicity up to 8-fold more potent than SAHA in three human cancer cell lines, including SW620 (colon cancer), PC3 (prostate cancer) and AsPC-1 (pancreatic cancer). A molecular modeling approach has been carried out which revealed some structure-activity relationships. Further investigation on absorption, distribution, metabolism, excretion and toxicity (ADMET) suggested that compounds $\mathbf{5 e}$ and $\mathbf{5 f}$, while showing potent HDAC2 inhibitory bioactivity, hold desirable characteristics for anticancer compounds.
\end{abstract}

Keywords. Histonedeacetylase (HDAC) inhibitors; hydroxamic acids; 2-oxoindoline; triazole.

\section{Introduction}

Target-based drug design and discovery has become a main stream in today's new drug discovery and development. In the field of cancer research, thanks to the recent advances in molecular pathology, hundreds of proteins have been validated as potential molecular targets for anticancer drug design. These include protein kinases, farnesyltransferases, telomerases, and histone deacetylases, among many others. ${ }^{1}$

Histone deacetylases (HDAC) are a group of enzymes catalyzing removal of the acetyl groups from lysine residues in the tails of histone proteins. ${ }^{2,3}$ Hitherto, 18 different isoforms of HDACs have been identified in eukaryotes and these isoforms are categorized

\footnotetext{
*For correspondence
}

into four classes based on the relative sequence similarity. ${ }^{2-4}$ Among these four classes, class I of HDACs, which has four members (HDAC1, 2, 3 and HDAC8) and class II, which has six members (HDAC4, 5, 6, 7, 9 and HDAC10) have been comprehensively investigated and demonstrated to be deeply involved in a number of cell-related processes. ${ }^{3-5}$ Specifically, HDAC 1, 2, 3 and 8 have been shown to promote cellular proliferation, meanwhile HDAC 1-4, 5 and 8 prevent cellular apoptosis and differentiation. Other HDAC isoforms, for example, HDAC 4, 6, 7 and 10, have been demonstrated to promote angiogenesis and cell migration, two processes very important for cancer cell metastasis. ${ }^{4,5}$ Down-regulation of these HDAC isoforms has been shown to result in a number of events related to differentiation, apoptosis and cell cycle arrest in different types of tumor cells. ${ }^{6}$ Moreover, selective 


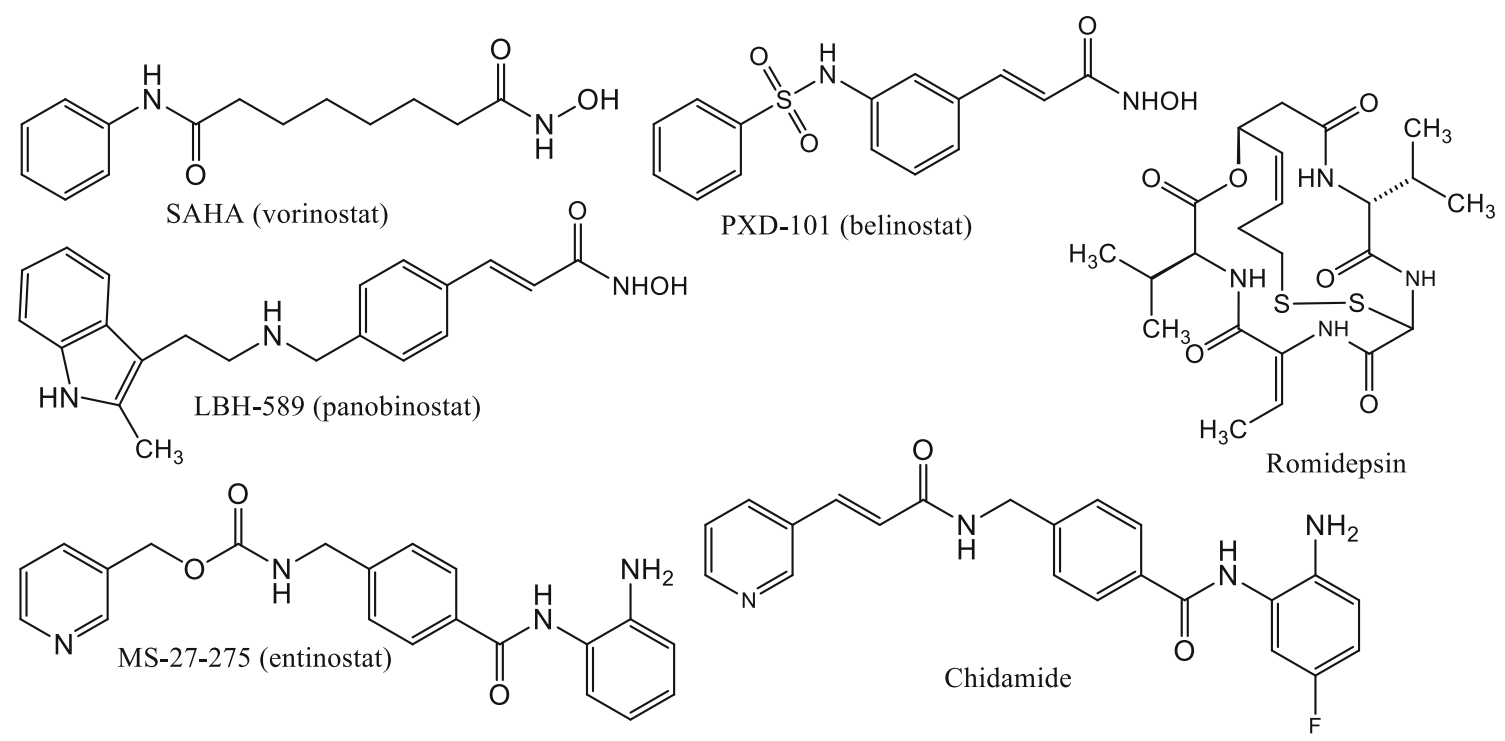

Figure 1. Structures of some HDAC inhibitors.

suppression of the growth of tumor cells caused by HDAC inhibition has been clearly demonstrated not only in vitro but also in a number of in vivo preclinical models and clinical settings. ${ }^{6}$ Design of compounds to inhibit appropriate HDACs has, therefore, become a very interesting approach in cancer drug design and development nowadays. ${ }^{7}$ As a result, a number of HDAC inhibitors have been reported in the past decades. These inhibitors are diverse, from short-chain fatty acids (like butyrate, phenylbutyrate or valproic acid) to hydroxamic acids, or benzamides. ${ }^{8-14}$ To date, at least 4 HDAC inhibitors have been approved for use in clinical settings. The first HDAC inhibitor approved by the U.S. FDA in October 2006 for the treatment of cutaneous $\mathrm{T}$ cell lymphoma (CTCL) was vorinostat (trade name, Zolinza ${ }^{\circledR}$ ) (also known as SAHA or suberoylanilidehydroxamic acid) (Figure 1). In 2009, the second HDAC inhibitor, romidepsin (trade name, Istodax ${ }^{\circledR}$ ) was also approved by the U.S. FDA for the same indication. In Feb 2015, panobinostat (LBH-589, trade name Farydak $($ ) ) was licensed by the US FDA for the treatment of multiple myeloma. ${ }^{15}$ Also in 2015 chidamide (Epidaza $\left.{ }^{\circledR}\right)$ was approved by the Chinese FDA for relapsed or refractory peripheral T cell lymphoma. ${ }^{16}$ In addition, a number of other HDAC inhibitors such as PXD-01 (belinostat), MS-27-527 (entinostat) (Figure 1) are currently under different phases of clinical trials for several types of cancer (Figure 1).

In continuation of our research program to develop novel hydroxamic acids as potential inhibitors of HDACs and anticancer agents, we have designed, synthesized and evaluated several series of heterocyclic analogues of SAHA, which incorporated benzothiazole or 5-aryl-1,3,4-thiadiazole systems (Figure 2). These compounds exhibited very potent HDAC inhibitory activity as well as cytotoxicity. ${ }^{17-20}$ Several representative compounds from these series also demonstrated very potent antitumor activity in $\mathrm{PC}-3$ prostate cancer cells xenografted mice model. ${ }^{18}$ Encouraged by these results, we expanded our design to the new series of 17 hydroxamic acids. In these hydroxamic acids the 2-oxoindoline system is employed as a cap group and linkers of different lengths incorporating a triazole moiety are probed. The current paper reports the results we obtained from the synthesis, biological evaluation and computational study on these novel hydroxamic acids.

\section{Experimental}

\subsection{Chemistry}

2.1a Chemicals and instruments: Thin layer chromatography (TLC) was performed using Whatman ${ }^{\circledR} 250 \mu \mathrm{m}$ Silica Gel GF Uniplates and visualized under UV light at 254 and $365 \mathrm{~nm}$. TLC was used to check the progress of reactions and preliminary evaluation of compounds' homogeneity. Melting points were measured using a Gallenkamp Melting Point Apparatus (LabMerchant, London, United Kingdom) and are uncorrected. Purification of compounds was carried out using crystallization methods and/or open silica gel column flash chromatography employing Merck silica gel 60 (240 to $400 \mathrm{mesh}$ ) as stationary phase. Nuclear magnetic resonance spectra $\left({ }^{1} \mathrm{H}\right.$ NMR $)$ were recorded on a Bruker $500 \mathrm{MHz}$ spectrometer with DMSO- $d_{6}$ as solvent unless otherwise 


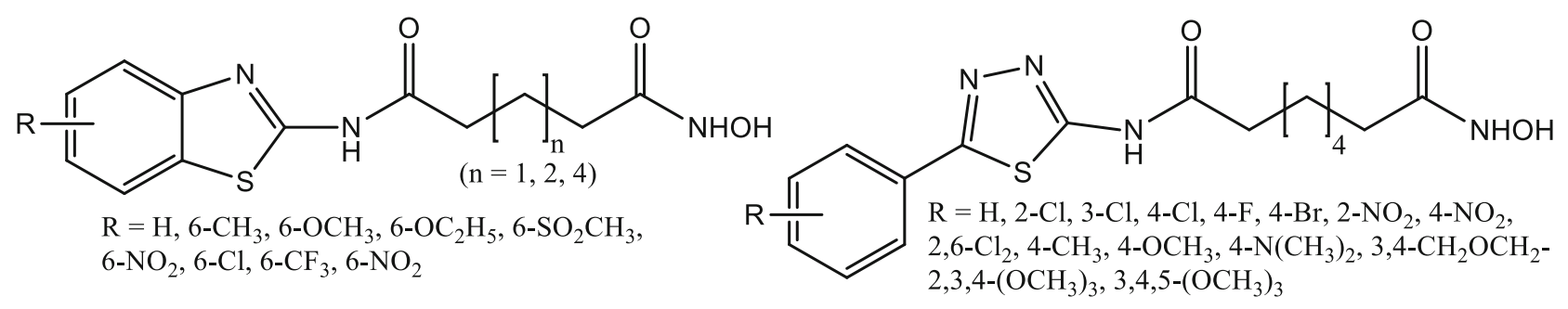

Figure 2. Structures of some benzothiazole- and 5-substituted phenyl-1,3,4-thiadiazole-based hydroxamic acids.

indicated. Tetramethylsilane was used as an internal standard. Chemical shifts are reported in parts per million (ppm), downfield from tetramethylsilane. The elemental $(\mathrm{C}, \mathrm{H}, \mathrm{N})$ analyses were performed on a Perkin Elmer model 2400 elemental analyzer. IR spectra were recorded on a Perkin Elmer FTIR model RX1 spectrometer ( $\mathrm{KBr}$ disc, $4000-400 \mathrm{~cm}^{-1}$ ). Mass spectra with different ionization modes including electron ionization (EI), Electrospray ionization (ESI), were recorded using PE Biosystems API2000 (Perkin Elmer, Palo Alto, CA, USA) and Mariner® (Azco Biotech, Inc.Oceanside, CA, USA) mass spectrometers, respectively. All the reagents (of synthetic grade with purity of $97 \%$ minimum) and solvents (of technical grade) were purchased from Aldrich, Fluka Chemical Corp. (Milwaukee, WI, USA) or Merck unless noted otherwise. Solvents were used directly as purchased unless otherwise indicated.

2.1b General procedures for the synthesis of compounds 4-6: The synthesis of the series of $17 \mathrm{~N}$ hydroxyalkanamides incorporating 1-((1H-1,2,3-triazol-4yl)methyl)-3-hydroxyiminoindolin-2-one moities (4-6) was carried out as illustrated in Scheme 1. Details are as follows:

To the respective solution of isatins $\mathbf{1 a}-\mathbf{g}(1 \mathrm{mmol})$ in DMF ( $3 \mathrm{~mL}$ ) were added $\mathrm{K}_{2} \mathrm{CO}_{3}(165.5 \mathrm{mg}, 1.2 \mathrm{mmol})$. The resulting mixtures were stirred at $80^{\circ} \mathrm{C}$ for $1 \mathrm{~h}$, then $\mathrm{KI}(8.3 \mathrm{mg}$, $0.05 \mathrm{mmol}$ ) was added. After stirring for further $15 \mathrm{~min}$, $0.15 \mathrm{~mL}$ of a solution of propargyl bromide $80 \%$ in toluene was added dropwise slowly into the mixtures. The reaction mixtures were again stirred at $60{ }^{\circ} \mathrm{C}$ for $3 \mathrm{~h}$. Upon completion of the reaction, the resulting mixtures were cooled, poured into ice-cold water and acidified to $\mathrm{pH} \sim 4$. The orange solids formed were filtered and dried to give the propargylatedisatins 2, which were used for the next step without further purification.

A respective solution of compounds 2 and methyl azidoesters $(1 \mathrm{mmol})$ in acetonitrile $(2 \mathrm{~mL})$ was stirred at room temperature for $10 \mathrm{~min}$, then $\mathrm{CuI}(19.1 \mathrm{mg}, 0.1 \mathrm{mmol})$ was added. The mixture was stirred at $50{ }^{\circ} \mathrm{C}$ until the reaction completed (12-24 h). The corresponding resulting mixtures were evaporated under reduced pressure to give the residues, which were re-dissolved in $50 \mathrm{~mL}$ of DCM. The mixtures were filtered and the DCM layers were evaporated under reduced pressure to give the intermediate esters $\mathbf{3}$, which were dissolved in a mixture of methanol and tetrahydrofuran $(2 / 1,5 \mathrm{~mL})$. Then, hydroxylamine. $\mathrm{HCl}(685 \mathrm{mg}, 10 \mathrm{mmol})$ was added, followed by dropwise addition of a solution of
$\mathrm{NaOH}$ (400 mg in $1 \mathrm{~mL}$ of water). The mixtures were stirred at room temperature until the reaction completed. The resulting reaction mixtures were poured into ice-cold water, neutralised to $\mathrm{pH} \sim 7$ and acidified by dropwise addition of a solution of $\mathrm{HCl} 5 \%$ to induce precipitation. The precipitates were filtered, dried and re-crystallized in methanol to give the target compounds 4-6.

\subsection{2a N-Hydroxy-3-(4-((3-(hydroxyimino)-2-oxoindo-} lin-1-yl)methyl)-1H-1,2,3-triazol-1-yl)propanamide

(4a): Yellow solid; Yield: $64 \%$. M.p.: $185.5-186.0^{\circ} \mathrm{C} . R_{f}$ $=0.40(\mathrm{DCM}: \mathrm{MeOH}: \mathrm{AcOH}=90: 5: 1) . I R\left(K B r, \mathrm{~cm}^{-1}\right)$ : $3423(\mathrm{NH}), 3287,3197(\mathrm{OH}), 3064(\mathrm{C}-\mathrm{H}$, aren), $2895(\mathrm{CH}$, $\left.\mathrm{CH}_{2}\right), 1723,1672(\mathrm{C}=\mathrm{O}), 1609,1549(\mathrm{C}=\mathrm{C}), 1462(\mathrm{C}-\mathrm{N}){ }^{1}$ $H$-NMR (500 MHz, DMSO-d 6 , ppm): $\delta 10.47(1 \mathrm{H}, \mathrm{s}, \mathrm{NH})$; $8.82(1 \mathrm{H}, \mathrm{s}, \mathrm{OH}) ; 8.02(1 \mathrm{H}, \mathrm{s}, \mathrm{H}-5$ ') $7.99(1 \mathrm{H}, \mathrm{d}, J=7.0 \mathrm{~Hz}$, H-4"); 7.39 (1H, t, $J=8.0 \mathrm{~Hz}, \mathrm{H}-6 ") ; 7.11(1 \mathrm{H}, \mathrm{t}, J=8.5$ Hz, H-5"); 7.07 (1H, d, J = 7.5 Hz, H-7'); 4.96 (2H, s, H-6'a, H-6'b); 4.51 (2H, t, $J=7.0$ Hz, H-3a, H-3b); 2.57 (2H, t, $J=$ $7.0 \mathrm{~Hz}, \mathrm{H}-2 \mathrm{a}, \mathrm{H}-2 \mathrm{~b}) .{ }^{13} \mathrm{C} \mathrm{NMR}\left(125 \mathrm{MHz}, \mathrm{DMSO}-d_{6}, \mathrm{ppm}\right)$ : $\delta$ 165.82, 162.75, 143.39, 142.54, 141.69, 131.88, 126.77, $123.51,122.69,115.27,109.62,45.58,34.57,32.49$. HR-MS (ESI) $m / z$ calculated for $\mathrm{C}_{14} \mathrm{H}_{14} \mathrm{~N}_{6} \mathrm{O}_{4}[\mathrm{M}+\mathrm{Na}]^{+} 353.0969$. Found 353.1001 .

2.1.2b 3-(4-((5-Fluoro-3-(hydroxyimino)-2-oxoindolin1-yl)methyl)-1H-1,2,3-triazol-1-yl)-N-hydroxypropanamide (4b): Yellow solid; Yield: 63\%. M.p.: 187.5-188.5 ${ }^{\circ} \mathrm{C} . R_{f}=0.42(\mathrm{DCM}: \mathrm{MeOH}: \mathrm{AcOH}=90: 5: 1) . I R(\mathrm{KBr}$, $\left.\mathrm{cm}^{-1}\right)$ : $3293(\mathrm{OH}), 3069(\mathrm{C}-\mathrm{H}$, aren $), 2921,2856\left(\mathrm{CH}, \mathrm{CH}_{2}\right)$, $1719(\mathrm{C}=\mathrm{O}), 1616(\mathrm{C}=\mathrm{C}), 1480,1439(\mathrm{C}-\mathrm{N}) .{ }^{1} H-N M R(500$ MHz, DMSO-d, ppm $): \delta 10.38(1 \mathrm{H}, \mathrm{s}, \mathrm{NH}) ; 8.05(1 \mathrm{H}, \mathrm{s}, \mathrm{H}-$ 5'); 7.74 (1H, dd, $J=8.0 \mathrm{~Hz}, J^{\prime}=2.0 \mathrm{~Hz}, \mathrm{H}-4$ '); 7.28 (1H, td, $\left.J=8.5 \mathrm{~Hz}, J^{\prime}=2.0 \mathrm{~Hz}, \mathrm{H}-6 ”\right) ; 7.13\left(1 \mathrm{H}, \mathrm{dd}, J=8.5 \mathrm{~Hz}, J^{\prime}\right.$ = 4.0 Hz, H-7'); 4.97 (2H, s, H-6'a, H-6'b); 4.53-4.48 (2H, m, H-3a, H-3b); 2.86 (1H, t, $J=6.75 \mathrm{~Hz}, \mathrm{H}-2 \mathrm{a}) 2.57$ (2H, t, $J=6.75 \mathrm{~Hz}, \mathrm{H}-2 \mathrm{~b}) .{ }^{13} \mathrm{CNMR}\left(125 \mathrm{MHz}, \mathrm{DMSO}-\mathrm{d}_{6}, \mathrm{ppm}\right)$ : $\delta 165.85,162.64,157.06,143.07,141.55,138.90,123.62$, 118.15, 115.77, 113.78, 110.77, 45.65, 34.72, 32.55. HR-MS (ESI) $m / z$ calculated for $\mathrm{C}_{14} \mathrm{H}_{13} \mathrm{FN}_{6} \mathrm{O}_{4}[\mathrm{M}-\mathrm{H}]^{-} 347.0909$. Found 347.0897 .

\subsection{2 c 3-(4-((5-Chloro-3-(hydroxyimino)-2-oxoindolin- 1-yl)methyl)-1H-1,2,3-triazol-1-yl)-N-hydroxypropan- amide (4c): Yellow solid; Yield: 63\%. M.p.: 187.0-189.0 ${ }^{\circ} \mathrm{C} . R_{f}=0.41(\mathrm{DCM}: \mathrm{MeOH}: \mathrm{AcOH}=90: 5: 1) . I R(\mathrm{KBr}$,}




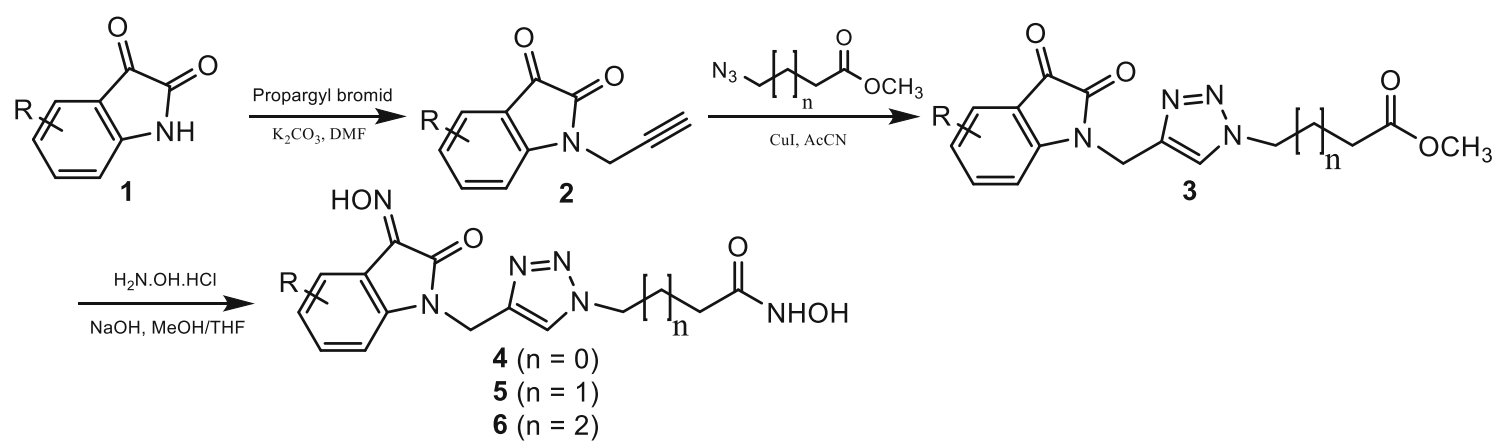

Scheme 1. Synthesis of hydroxamic acids incorporating 1-((1H-1,2,3-triazol-4-yl)methyl)-3-hydroxyiminoindolin-2-ones (4-6).

$\left.\mathrm{cm}^{-1}\right): 3420(\mathrm{NH}), 3255,3151(\mathrm{OH}), 3035(\mathrm{C}-\mathrm{H}$, aren $), 2851$ $\left(\mathrm{CH}, \mathrm{CH}_{2}\right), 1713,1647(\mathrm{C}=\mathrm{O}), 1609(\mathrm{C}=\mathrm{C}), 1464(\mathrm{C}-\mathrm{N}){ }^{1}$ $H$-NMR $\left(500 \mathrm{MHz}, \mathrm{DMSO}-\mathrm{d}_{6}, \mathrm{ppm}\right): \delta 14.70(1 \mathrm{H}, \mathrm{s}, \mathrm{NH})$; $10.48(1 \mathrm{H}, \mathrm{s}, \mathrm{NH}) ; 8.83(1 \mathrm{H}, \mathrm{s}, \mathrm{OH}) ; 8.03$ (1H, s, H-5'); 7.96 (1H, d, $J=1.75 \mathrm{~Hz}, \mathrm{H}-4 ") ; 7.48$ (1H, dd, $J=8.5 \mathrm{~Hz}$, $\left.J^{\prime}=1.75 \mathrm{~Hz}, \mathrm{H}-6 ”\right) ; 7.14$ (1H, d, $J=8.5 \mathrm{~Hz}, \mathrm{H}-7$ '”); 4.98 (2H, s, H-6'a, H-6'b); 4.52 (2H, t, J = 6.75 Hz, H-3a, H-3b); $2.57(2 \mathrm{H}, \mathrm{t}, J=6.75 \mathrm{~Hz}, \mathrm{H}-2 \mathrm{a}, \mathrm{H}-2 \mathrm{~b}) .{ }^{13} \mathrm{C} \mathrm{NMR}(125 \mathrm{MHz}$, DMSO- $d_{6}$, ppm): $\delta 165.83,162.40,142.66,141.43,141.31$, 131.37, 126.55, 126.08, 123.59, 116.40, 111.27, 45.62, 34.72, 32.51. HR-MS (ESI) $m / z$ calculated for $\mathrm{C}_{14} \mathrm{H}_{13} \mathrm{ClN}_{6} \mathrm{O}_{4}$, [M $+\mathrm{H}]^{+}$365.0760. Found, 365.0754.

2.1.2dN-Hydroxy-4-(4-((3-(hydroxyimino)-2-oxoindolin-1-yl)methyl)-1H-1,2,3-triazol-1-yl)butanamide (5a): Yellow solid; Yield: $68 \%$. M.p.: $181.0-182.5{ }^{\circ} \mathrm{C} . R_{f}=0.45$ (DCM : $\mathrm{MeOH}: \mathrm{AcOH}=90: 5: 1) . I R\left(\mathrm{KBr}, \mathrm{cm}^{-1}\right)$ : $3135(\mathrm{OH}), 3022$ (C-H, aren), 2918, $2856\left(\mathrm{CH}, \mathrm{CH}_{2}\right), 1721$, $1640(\mathrm{C}=\mathrm{O}), 1608(\mathrm{C}=\mathrm{C}), 1463(\mathrm{C}-\mathrm{N}) .{ }^{1} \mathrm{H}-\mathrm{NMR}(500 \mathrm{MHz}$, DMSO-d, ppm): $\delta 13.58(1 \mathrm{H}, \mathrm{s}, \mathrm{NH}) ; 10.52(1 \mathrm{H}, \mathrm{s}, \mathrm{OH})$; 10.40 (1H, s, NH); $8.12\left(1 \mathrm{H}, \mathrm{s}, \mathrm{H}-5^{\prime}\right) ; 7.97(1 \mathrm{H}, \mathrm{d}, J=7.5 \mathrm{~Hz}$, H-4"); 7.39 (1H, td, $\left.J=8.0 \mathrm{~Hz}, J^{\prime}=1.0 \mathrm{~Hz}, \mathrm{H}-6 "\right) ; 7.11(1 \mathrm{H}$, d, $J=7.5 \mathrm{~Hz}, \mathrm{H}-7$ '); 7.07 (1H, t, $J=7.5 \mathrm{~Hz}, \mathrm{H}-5$ '); 4.97 (2H, s, H-6'a, H-6'b); 4.29 (2H, t, $J=6.5$ Hz, H-4a, H-4b); 1.991.95 (4H, m, H-3a, H-3b, H-2a, H-2b). ${ }^{13} \mathrm{C} \mathrm{NMR}(125 \mathrm{MHz}$, DMSO- $d_{6}$, ppm): $\delta 168.16,162.83,143.36,142.60,141.87$, 131.94, 126.83, 123.39, 122.77, 115.32, 109.68, 48.93, 34.67, 29.01, 25.85.MS (ESI) $m / z 343.12[\mathrm{M}-\mathrm{H}]^{-}$. Anal. Calcd. For $\mathrm{C}_{15} \mathrm{H}_{16} \mathrm{~N}_{6} \mathrm{O}_{4}$ (344.33): C, 52.32; H, 4.68; N, 24.41. Found: C, 52.37; H, 4.71; N, 24.38.

2.1.2e 4-(4-((5-Fluoro-3-(hydroxyimino)-2-oxoindolin1-yl)methyl)-1H-1,2,3-triazol-1-yl)-N-hydroxybutanamide (5b): Yellow solid; Yield: 72\%. M.p.: 180.0-182.0 ${ }^{\circ} \mathrm{C} . R_{f}=0.43(\mathrm{DCM}: \mathrm{MeOH}: \mathrm{AcOH}=90: 5: 1) . I R$ $\left(\mathrm{KBr}, \mathrm{cm}^{-1}\right)$ : $3441(\mathrm{NH}), 3208,3137(\mathrm{OH}), 3029$ (C-H, aren), $2872\left(\mathrm{CH}, \mathrm{CH}_{2}\right), 1722,1640(\mathrm{C}=\mathrm{O}), 1477(\mathrm{C}-\mathrm{N}) .{ }^{1} H-N M R$ (500 MHz, DMSO-d 6 , ppm): $\delta 10.40(1 \mathrm{H}, \mathrm{s}, \mathrm{NH}) ; 8.72$ $(1 \mathrm{H}, \mathrm{s}, \mathrm{OH}) ; 8.11(1 \mathrm{H}, \mathrm{s}, \mathrm{H}-5$ ' $) ; 7.75(1 \mathrm{H}, \mathrm{dd}, J=7.5 \mathrm{~Hz}$, $\left.J^{\prime}=2.5 \mathrm{~Hz}, \mathrm{H}-4 ”\right) ; 7.28\left(1 \mathrm{H}, \mathrm{td}, J=8.5 \mathrm{~Hz}, J^{\prime}=2.5\right.$ Hz, H-6"); 7.13 (1H, dd, $J=8.5 \mathrm{~Hz}, J^{\prime}=4.0 \mathrm{~Hz}, \mathrm{H}-7$ ');
4.98 (2H, s, H-6'a, H-6'b); 4.32-4.29 (2H, m, H-4a, H-4b); 1.99-1.92 (4H, m, H-3a, H-3b, H-2a, H-2b). ${ }^{13} C$ NMR (125 $\left.\mathrm{MHz}, \mathrm{DMSO}-\mathrm{d}_{6}, \mathrm{ppm}\right): \delta 168.05,162.64,157.06,143.13$, 141.72 , 138.92, 123.43, 118.14, 115.79, 113.80, 110.75,

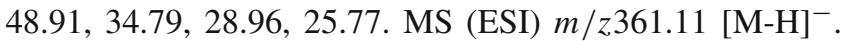
Anal. Calcd. For $\mathrm{C}_{15} \mathrm{H}_{15} \mathrm{FN}_{6} \mathrm{O}_{4}$ (362.32): C, 49.72; H, 4.17; N, 23.20. Found: C, 49.75; H, 4.21; N, 23.24.

2.1.2f 4-(4-((5-Chloro-3-(hydroxyimino)-2-oxoindolin1-yl)methyl)-1H-1,2,3-triazol-1-yl)-N-hydroxybutanamide (5c): Yellow solid; Yield: 64\%. M.p.: $187.5-188.5^{\circ} \mathrm{C}$. $R_{f}=0.42(\mathrm{DCM}: \mathrm{MeOH}: \mathrm{AcOH}=90: 5: 1) . I R(K B r$, $\left.\mathrm{cm}^{-1}\right): 3417(\mathrm{NH}), 3219,3138(\mathrm{OH}), 3044(\mathrm{C}-\mathrm{H}$, aren $), 2874$ $\left(\mathrm{CH}, \mathrm{CH}_{2}\right), 1720,1643(\mathrm{C}=\mathrm{O}), 1610(\mathrm{C}=\mathrm{C}), 1463(\mathrm{C}-\mathrm{N}){ }^{1}$ $H-N M R\left(500 \mathrm{MHz}, \mathrm{DMSO}-d_{6}, \mathrm{ppm}\right): \delta 13.82(1 \mathrm{H}, \mathrm{s}, \mathrm{NH})$; $10.38(1 \mathrm{H}, \mathrm{s}, \mathrm{NH}) ; 8.71(1 \mathrm{H}, \mathrm{s}, \mathrm{OH}) ; 8.10(1 \mathrm{H}, \mathrm{s}, \mathrm{H}-5$ ') 7.96 $\left(1 \mathrm{H}, \mathrm{d}, J=1.75 \mathrm{~Hz}, \mathrm{H}-4\right.$ "); $7.47\left(1 \mathrm{H}, \mathrm{dd}, J=7.5 \mathrm{~Hz}, J^{\prime}=\right.$ $1.75 \mathrm{~Hz}, \mathrm{H}-6 ") ; 7.14$ (1H, d, $J=8.5 \mathrm{~Hz}, \mathrm{H}-7$ "); 4.98 (2H, s, H-6'a, H-6'b); 4.30 (2H, t, $J=6.5 \mathrm{~Hz}, \mathrm{H}-4 \mathrm{a}, \mathrm{H}-4 \mathrm{~b}) ; 2.00$ $1.92(4 \mathrm{H}, \mathrm{m}, \mathrm{H}-3 \mathrm{a}, \mathrm{H}-3 \mathrm{~b}, \mathrm{H}-2 \mathrm{a}, \mathrm{H}-2 \mathrm{~b}) .{ }^{13} \mathrm{C} N M R(125 \mathrm{MHz}$, DMSO-d, ppm): $\delta 168.05,162.41,142.71,141.63,141.38$, $131.40,126.56,126.12,123.33,116.43,111.28,48.92,34.82$, 28.96, 25.76. MS (ESI) $m / z 401.07[\mathrm{M}+\mathrm{Na}]^{+}$. Anal. Calcd. For $\mathrm{C}_{15} \mathrm{H}_{15} \mathrm{ClN}_{6} \mathrm{O}_{4}$ (378.77): C, 47.56; H, 3.99; N, 22.19 . Found: C, 47.61; H, 3.95; N, 22.22.

2.1.2g 4-(4-((5-Bromo-3-(hydroxyimino)-2-oxoindolin1-yl)methyl)-1H-1,2,3-triazol-1-yl)-N-hydroxybutanamide (5d): Yellow solid; Yield: 68\%. M.p.: 188.5-189.5 ${ }^{\circ} \mathrm{C} . R_{f}=0.47(\mathrm{DCM}: \mathrm{MeOH}: \mathrm{AcOH}=90: 5: 1) . I R$ $\left(\mathrm{KBr}, \mathrm{cm}^{-1}\right): 3407(\mathrm{NH}), 3138(\mathrm{OH}), 3023(\mathrm{C}-\mathrm{H}$, aren $), 2968$, $2853\left(\mathrm{CH}, \mathrm{CH}_{2}\right), 1731,1704(\mathrm{C}=\mathrm{O}), 1606(\mathrm{C}=\mathrm{C}), 1463(\mathrm{C}-$ N). ${ }^{1} \mathrm{H}-\mathrm{NMR}\left(500 \mathrm{MHz}, \mathrm{DMSO}-\mathrm{d}_{6}, \mathrm{ppm}\right): \delta 10.42(1 \mathrm{H}$, s, NH); $8.72(1 \mathrm{H}, \mathrm{s}, \mathrm{OH}) ; 8.10(1 \mathrm{H}, \mathrm{s}, \mathrm{H}-5$ '); $8.08(1 \mathrm{H}, \mathrm{d}$, $J=1.5 \mathrm{~Hz}, \mathrm{H}-4$ "); $7.60\left(1 \mathrm{H}, \mathrm{dd}, J=8.25 \mathrm{~Hz}, J^{\prime}=1.5\right.$ Hz, H-6"); 7.10 (1H, d, $J=8.25 \mathrm{~Hz}, \mathrm{H}-7 ”) ; 4.98(2 \mathrm{H}, \mathrm{s}$, H-6'a, H-6'b); 4.30 (2H, t, $J=6.5$ Hz, H-4a, H-4b); 2.001.94 (4H, m, H-3a, H-3b, H-2a, H-2b). ${ }^{13} \mathrm{C} N M R(125 \mathrm{MHz}$, DMSO-d $d_{6}$, ppm): $\delta 168.07,162,34,142.52,141.72,141.60$, 134.17, 128.76, 123.35, 116.88, 114.20, 111.78, 48.92, 34.79, 28.97, 25.78.MS (ESI) $m / z 421.10\left[^{\mathrm{M}-\mathrm{H}}\right]^{-}{ }^{-}$. Anal. Calcd. For 
$\mathrm{C}_{15} \mathrm{H}_{15} \mathrm{BrN}_{6} \mathrm{O}_{4}$ (422.23): $\mathrm{C}, 42.57 ; \mathrm{H}, 3.57 ; \mathrm{N}, 19.86$. Found: C, 42.59; H, 3.61; N, 19.85 .

2.1.2hN-Hydroxy-4-(4-((3-(hydroxyimino)-5-methyl-2oxoindolin-1-yl)methyl)-1H-1,2,3-triazol-1-yl)butanamide (5e): Yellow solid; Yield: $71 \%$. M.p.: $179.5-181.0{ }^{\circ} \mathrm{C}$. $R_{f}=0.41(\mathrm{DCM}: \mathrm{MeOH}: \mathrm{AcOH}=90: 5: 1) . I R(K B r$, $\left.\mathrm{cm}^{-1}\right): 3280(\mathrm{NH}), 3184(\mathrm{OH}), 3068(\mathrm{C}-\mathrm{H}$, aren $), 2908(\mathrm{CH}$, $\left.\mathrm{CH}_{2}\right), 1713,1673(\mathrm{C}=\mathrm{O}), 1624,1552(\mathrm{C}=\mathrm{C}), 1476(\mathrm{C}-\mathrm{N}) .{ }^{1}$ $H-N M R\left(500 \mathrm{MHz}, \mathrm{DMSO}-d_{6}, \mathrm{ppm}\right): \delta 13.44(1 \mathrm{H}, \mathrm{s}, \mathrm{NH})$; $10.38(1 \mathrm{H}, \mathrm{s}, \mathrm{NH}) ; 8.71(1 \mathrm{H}, \mathrm{s}, \mathrm{OH}) ; 8.08\left(1 \mathrm{H}, \mathrm{s}, \mathrm{H}-5^{\prime}\right)$; 7.83 (1H, s, H-4"); 7.20 (1H, d, $J=8.0 \mathrm{~Hz}, \mathrm{H}-7$ "); 6.99 $(1 \mathrm{H}, \mathrm{d}, J=8.0 \mathrm{~Hz}, \mathrm{H}-6$ '); 4.95 (2H, s, H-6'a, H-6'b); $4.30(2 \mathrm{H}, \mathrm{t}, J=6.5 \mathrm{~Hz}, \mathrm{H}-4 \mathrm{a}, \mathrm{H}-4 \mathrm{~b}) ; 2.27\left(3 \mathrm{H}, \mathrm{s},-\mathrm{CH}_{3}\right)$; 1.98-1.93 (4H, m, H-3a, H-3b, H-2a, H-2b). ${ }^{13} C$ NMR (125 $\left.M H z, D M S O-d_{6}, p p m\right): \delta 168.08,162.77,143.59,141.93$, $140.39,132.14,131.77,127.38,123.26,115.35,109.42$, $48.89,34.67,28.97,25.78,20.51$. HR-MS (ESI) $m / z$ calculated for $\mathrm{C}_{16} \mathrm{H}_{18} \mathrm{~N}_{6} \mathrm{O}_{4}[\mathrm{M}+\mathrm{Na}]^{+}$381.1282. Found 381.1270.

2.1.2i N-Hydroxy-4-(4-((3-(hydroxyimino)-5-methoxy2-oxoindolin-1-yl)methyl)-1H-1,2,3-triazol-1-yl)butanamide (5f): Yellow solid; Yield: 60\%. M.p.: $180.0-182.0^{\circ} \mathrm{C}$. $R_{f}=0.45(\mathrm{DCM}: \mathrm{MeOH}: \mathrm{AcOH}=90: 5: 1) . I R\left(\mathrm{KBr}, \mathrm{cm}^{-1}\right)$ : $3281(\mathrm{NH}), 3187(\mathrm{OH}), 3071(\mathrm{C}-\mathrm{H}$, aren $), 2916\left(\mathrm{CH}, \mathrm{CH}_{2}\right)$, $1711,1673(\mathrm{C}=\mathrm{O}), 1630,1596(\mathrm{C}=\mathrm{C}), 1552,1480(\mathrm{C}-\mathrm{N}) .{ }^{1} \mathrm{H}$ NMR $\left(500 \mathrm{MHz}, \mathrm{DMSO}-d_{6}, \mathrm{ppm}\right): \delta 13.53(1 \mathrm{H}, \mathrm{s}, \mathrm{NH}) ; 10.40$ $(1 \mathrm{H}, \mathrm{s}, \mathrm{NH}) ; 8.09\left(1 \mathrm{H}, \mathrm{s}, \mathrm{H}-5^{\prime}\right) ; 7.58$ (1H, d, $J=2.5 \mathrm{~Hz}, \mathrm{H}-4$ '); 7.04-6.98 (2H, m, H-6",H-7'); 4.94 (2H, s, H-6'a, H-6'b); $4.30(2 \mathrm{H}, \mathrm{t}, J=7.0 \mathrm{~Hz}, \mathrm{H}-4 \mathrm{a}, \mathrm{H}-4 \mathrm{~b}) ; 3.72\left(3 \mathrm{H}, \mathrm{s},-\mathrm{OCH}_{3}\right)$; 2.00-1.92 (4H, m, H-3a, H-3b, H-2a, H-2b). ${ }^{13}$ C NMR (125 MHz, DMSO-d 6 , ppm): $\delta 168.05,162.62,155.21,143.68$, $141.94,136.24,123.28,116.86,115.85,113.09,110.26$, $55.65,48.90,34.70,28.97,25.78$. HR-MS (ESI) $\mathrm{m} / z$ calculated for $\mathrm{C}_{16} \mathrm{H}_{18} \mathrm{~N}_{6} \mathrm{O}_{5}[\mathrm{M}-\mathrm{H}]^{-}$373.1266. Found 373.1241.

2.1.2j 4-(4-((7-Chloro-3-(hydroxyimino)-2-oxoindolin1-yl)methyl)-1H-1,2,3-triazol-1-yl)-N-hydroxybutanamide (5g): Yellow solid; Yield: $70 \%$. M.p.: $187.5-188.5^{\circ} \mathrm{C}$. $R_{f}=0.43$ (DCM : MeOH : $\left.\mathrm{AcOH}=90: 5: 1\right) . I R(K B r$, $\left.\mathrm{cm}^{-1}\right)$ : 3517, $3378(\mathrm{NH}), 3248(\mathrm{OH}), 3030(\mathrm{C}-\mathrm{H}$, aren $), 2897$ $\left(\mathrm{CH}, \mathrm{CH}_{2}\right), 1717,1664(\mathrm{C}=\mathrm{O}), 1634,1608(\mathrm{C}=\mathrm{C}), 1441(\mathrm{C}-$ N). ${ }^{1} H-N M R\left(500 \mathrm{MHz}, D M S O-d_{6}, p p m\right): \delta 10.41(1 \mathrm{H}, \mathrm{s}$, $\mathrm{NH}) ; 8.72(1 \mathrm{H}, \mathrm{s}, \mathrm{OH}) ; 8.07\left(1 \mathrm{H}, \mathrm{dd}, J=7.75 \mathrm{~Hz}, J^{\prime}=1.0\right.$ Hz, H-4"); 8.06 (1H, s, H-5'); 7.39 (1H, dd, $J=7.75 \mathrm{~Hz}$, $J^{\prime}=1.0 \mathrm{~Hz}, \mathrm{H}-6$ "); $7.11(1 \mathrm{H}, \mathrm{t}, J=7.75 \mathrm{~Hz}, \mathrm{H}-5$ "); 5.31 (2H, s, H-6'a, H-6'b); 4.29 (2H, t, $J=6.5 \mathrm{~Hz}, \mathrm{H}-4 \mathrm{a}, \mathrm{H}-4 \mathrm{~b})$; 2.00-1.92 (4H, m, H-3a, H-3b, H-2a, H-2b). ${ }^{13}$ C NMR (125 MHz, DMSO-d $\left.d_{6}, \mathrm{ppm}\right): \delta 168.07,163.64,143.38,142.19$, $138.46,133.78,125.84,124.23,122.49,118.30,114.80$, $48.88,37.06,28.96,25.84$. HR-MS (ESI) $m / z$ calculated for $\mathrm{C}_{15} \mathrm{H}_{15} \mathrm{ClN}_{6} \mathrm{O}_{4}[\mathrm{M}-\mathrm{H}]^{-}$377.0770. Found 377.0748.

2.1.2k N-Hydroxy-5-(4-((3-(hydroxyimino)-2-oxoindolin-1-yl)methyl)-1H-1,2,3-triazol-1-yl)pentanamide (6a): Yellow solid; Yield: 68\%. M.p.: $189.0-191.5{ }^{\circ} \mathrm{C} . R_{f}$ $=0.48(\mathrm{DCM}: \mathrm{MeOH}: \mathrm{AcOH}=90: 5: 1) . I R\left(K B r, \mathrm{~cm}^{-1}\right)$ :
3399, $3312(\mathrm{NH}), 3196(\mathrm{OH}), 3059(\mathrm{C}-\mathrm{H}$, aren $), 2876(\mathrm{CH}$, $\left.\mathrm{CH}_{2}\right), 1714,1678(\mathrm{C}=\mathrm{O}), 1608(\mathrm{C}=\mathrm{C}), 1455(\mathrm{C}-\mathrm{N}) .{ }^{1} H-N M R$ $\left(500 \mathrm{MHz}, D M S O-d_{6}, p p m\right): \delta 13.45(1 \mathrm{H}, \mathrm{s}, \mathrm{NH}) ; 10.35(1 \mathrm{H}$, s, NH); $8.67(1 \mathrm{H}, \mathrm{s}, \mathrm{OH}) ; 8.09(1 \mathrm{H}, \mathrm{s}, \mathrm{H}-5$ '); $7.98(1 \mathrm{H}, \mathrm{d}, J=$ $7.5 \mathrm{~Hz}, \mathrm{H}-4$ "); 7.40 (1H, t, $J=7.5 \mathrm{~Hz}, \mathrm{H}-6 ") ; 7.12-7.06(2 \mathrm{H}$, m, H-5", H-7"); 4.98 (2H, s, H-6'a, H-6'b); 4.29 (2H, t, J = 7.0 Hz, H-5a, H-5b); 1.95 (2H, t, J = 7.5 Hz, H-2a, H-2b); 1.79 1.71 (2H, m, H-4a, H-4b); $1.45-1.39$ (2H, m, H-3a, H-3b). ${ }^{13} \mathrm{C}$ NMR $\left(125 \mathrm{MHz}, \mathrm{DMSO}-d_{6}, \mathrm{ppm}\right): \delta 168.68,162.74,143.44$, $142.61,141.80,131.95,126.85,123.26,122.73,115.28$, 109.64, 49.05, 34.67, 29.22, 22.04. HR-MS (ESI) $\mathrm{m} / \mathrm{z}$ calculated for $\mathrm{C}_{16} \mathrm{H}_{18} \mathrm{~N}_{6} \mathrm{O}_{4}[\mathrm{M}-\mathrm{H}]^{-}$357.1317. Found 357.1313.

2.1.2l 5-(4-((5-Fluoro-3-(hydroxyimino)-2-oxoindolin1-yl)methyl)-1H-1,2,3-triazol-1-yl)- $N$-hydroxypentanamide (6b): Yellow solid; Yield: 69\%. M.p.: $194.5-196.0^{\circ} \mathrm{C}$. $R_{f}=0.48(\mathrm{DCM}: \mathrm{MeOH}: \mathrm{AcOH}=90: 5: 1) . I R(\mathrm{KBr}$, $\left.\mathrm{cm}^{-1}\right): 3396,3320(\mathrm{NH}), 3172(\mathrm{OH}), 3058(\mathrm{C}-\mathrm{H}$, aren $), 2876$ $\left(\mathrm{CH}, \mathrm{CH}_{2}\right), 1715,1680(\mathrm{C}=\mathrm{O}), 1630(\mathrm{C}=\mathrm{C}), 1477(\mathrm{C}-\mathrm{N}){ }^{1}$ $H-N M R\left(500 \mathrm{MHz}, D M S O-d_{6}, p p m\right): \delta 13.76(1 \mathrm{H}, \mathrm{s}, \mathrm{NH})$; $10.34(1 \mathrm{H}, \mathrm{s}, \mathrm{NH}) ; 8.67(1 \mathrm{H}, \mathrm{s}, \mathrm{OH}) ; 8.08(1 \mathrm{H}, \mathrm{s}, \mathrm{H}-5$ '); 7.75 $\left(1 \mathrm{H}, \mathrm{dd}, J=8.0 \mathrm{~Hz}, J^{\prime}=2.5 \mathrm{~Hz}, \mathrm{H}-4\right.$ "); $7.28(1 \mathrm{H}, \mathrm{td}, J=$ $\left.8.75 \mathrm{~Hz}, J^{\prime}=2.5 \mathrm{~Hz}, \mathrm{H}-6^{\prime}\right) ; 7.12\left(1 \mathrm{H}, \mathrm{dd}, J=8.75 \mathrm{~Hz}, J^{\prime}=\right.$ 4.0 Hz, H-7"); 4.98 (2H, s, H-6' a, H-6'b); 4.29 (2H, t, $J=7.0$ Hz, H-5a, H-5b); 1.95 (2H, t, $J=7.5$ Hz, H-2a, H-2b); 1.761.73 (2H, m, H-4a, H-4b); 1.45-1.40 (2H, m, H-3a, H-3b). ${ }^{13} \mathrm{C}$ NMR $\left(125 \mathrm{MHz}, \mathrm{DMSO}-d_{6}, \mathrm{ppm}\right): \delta 168.68,162.61$, $158.00,143.16,141.64,138.93,123.29,118.15,115.77$, $113.83,110.72,49.06,34.79,31.51,29.19,22.03$. HR-MS (ESI) $m / z$ calculated for $\mathrm{C}_{16} \mathrm{H}_{17} \mathrm{FN}_{6} \mathrm{O}_{4}[\mathrm{M}-\mathrm{H}]^{-} 375.1222$. Found 375.1229.

\subsection{2m 5-(4-((5-Chloro-3-(hydroxyimino)-2-oxoindoli-} n-1-yl)methyl)-1H-1,2,3-triazol-1-yl)-N-hydroxypentanamide (6c): Yellow solid; Yield: 69\%. M.p.: 194.5-196.0 ${ }^{\circ} \mathrm{C} . R_{f}=0.48(\mathrm{DCM}: \mathrm{MeOH}: \mathrm{AcOH}=90: 5: 1) . I R(\mathrm{KBr}$, $\left.\mathrm{cm}^{-1}\right)$ : $3286(\mathrm{NH}), 3143(\mathrm{OH}), 3042(\mathrm{C}-\mathrm{H}$, aren $), 2851(\mathrm{CH}$, $\left.\mathrm{CH}_{2}\right), 1708,1647(\mathrm{C}=\mathrm{O}), 1608(\mathrm{C}=\mathrm{C}), 1461(\mathrm{C}-\mathrm{N}) .{ }^{1} \mathrm{H}-\mathrm{NMR}$ $\left(500 \mathrm{MHz}, \mathrm{DMSO}_{-} \mathrm{d}_{6}, \mathrm{ppm}\right): \delta 10.36(1 \mathrm{H}, \mathrm{s}, \mathrm{NH}) ; 8.68(1 \mathrm{H}$, s, OH); $8.08(1 \mathrm{H}, \mathrm{s}, \mathrm{H}-5$ '); $7.95(1 \mathrm{H}, \mathrm{s}, \mathrm{H}-4$ "); $7.48(1 \mathrm{H}, \mathrm{d}$, $J=8.0 \mathrm{~Hz}, \mathrm{H}-6 ”) ; 7.14(1 \mathrm{H}, \mathrm{d}, J=8.0 \mathrm{~Hz}, \mathrm{H}-7$ '); 4.98 (2H, s, H-6'a, H-6'b); 4.29 (2H, t, $J=7.0 \mathrm{~Hz}, \mathrm{H}-5 \mathrm{a}, \mathrm{H}-5 \mathrm{~b})$; $1.95(2 \mathrm{H}, \mathrm{t}, J=7.5 \mathrm{~Hz}, \mathrm{H}-2 \mathrm{a}, \mathrm{H}-2 \mathrm{~b}) ; 1.77-1.72(2 \mathrm{H}, \mathrm{m}, \mathrm{H}-$ 4a, H-4b); 1.45-1.40 (2H, m, H-3a, H-3b). ${ }^{13}$ C NMR (125 $\left.M H z, D M S O-d_{6}, p p m\right): \delta 168.73,162.44,142.71,141.57$, $141.38,131.42,126.59,126.14,123.31,116.44,111.29$, 49.09, 34.84, 31.53, 29.22, 22.06. HR-MS (ESI) $\mathrm{m} / \mathrm{z}$ calculated for $\mathrm{C}_{16} \mathrm{H}_{17} \mathrm{ClN}_{6} \mathrm{O}_{4}[\mathrm{M}-\mathrm{H}]^{-}$391.0927. Found 391.0938.

2.1.2n 5-(4-((5-Bromo-3-(hydroxyimino)-2-oxoindolin1-yl)methyl)-1H-1,2,3-triazol-1-yl)- $N$-hydroxypentanamide (6d): Yellow solid; Yield: 74\%. M.p.: 201.5-203.0 ${ }^{\circ} \mathrm{C}$. $R_{f}=0.51(\mathrm{DCM}: \mathrm{MeOH}: \mathrm{AcOH}=90: 5: 1) \cdot I R\left(\mathrm{KBr}, \mathrm{cm}^{-1}\right)$ : 3420, $3289(\mathrm{NH}), 3147(\mathrm{OH}), 3031(\mathrm{C}-\mathrm{H}$, aren), $2862(\mathrm{CH}$, $\left.\mathrm{CH}_{2}\right), 1708,1646(\mathrm{C}=\mathrm{O}), 1606(\mathrm{C}=\mathrm{C}), 1462(\mathrm{C}-\mathrm{N}) .{ }^{1} \mathrm{H}-\mathrm{NMR}$ $\left(500 \mathrm{MHz}, \mathrm{DMSO}-d_{6}, p p m\right): \delta 10.34(1 \mathrm{H}, \mathrm{s}, \mathrm{NH}) ; 8.67(1 \mathrm{H}$, s, OH); 8.08 (2H, s, H-5', H-4"); $7.60(1 \mathrm{H}, \mathrm{d}, J=7.75 \mathrm{~Hz}, \mathrm{H}-$ 
6"); 7.09 (1H, d, J = $7.75 \mathrm{~Hz}, \mathrm{H}-7$ "); 4.98 (2H, s, H-6'a, H-6’b); 4.29 (2H, t, $J=6.0 \mathrm{~Hz}, \mathrm{H}-5 \mathrm{a}, \mathrm{H}-5 \mathrm{~b}) ; 1.95(2 \mathrm{H}$, $\mathrm{t}, J=6.5 \mathrm{~Hz}, \mathrm{H}-2 \mathrm{a}, \mathrm{H}-2 \mathrm{~b}) ; 1.75-1.73(2 \mathrm{H}, \mathrm{m}, \mathrm{H}-4 \mathrm{a}, \mathrm{H}-$ 4b); 1.45-1.41 (2H, m, H-3a, H-3b). ${ }^{13} \mathrm{C} \mathrm{NMR} \mathrm{(125} \mathrm{MHz,}$ DMSO-d $d_{6}$, ppm): $\delta 168.68,162.31,142.57,141.73,141.54$, $134.20,128.79,123.27,116.86,114.21,111.76,49.07$, 34.80, 31.51, 29.20, 22.04. HR-MS (ESI) $\mathrm{m} / \mathrm{z}$ calculated for $\mathrm{C}_{16} \mathrm{H}_{17} \mathrm{BrN}_{6} \mathrm{O}_{4},[\mathrm{M}-\mathrm{H}]^{-}$437.0422. Found 437.0421.

2.1.2o N-Hydroxy-5-(4-((3-(hydroxyimino)-5-methyl-2oxoindolin-1-yl)methyl)-1H-1,2,3-triazol-1-yl)pentanamide (6e): Yellow solid; Yield: 75\%. M.p.: 199.5-201.6 ${ }^{\circ} \mathrm{C}$. $R_{f}=0.46(\mathrm{DCM}: \mathrm{MeOH}: \mathrm{AcOH}=90: 5: 1) . I R(K B r$, $\left.\mathrm{cm}^{-1}\right): 3317(\mathrm{NH}), 3188(\mathrm{OH}), 3061(\mathrm{C}-\mathrm{H}$, aren $), 2879(\mathrm{CH}$, $\left.\mathrm{CH}_{2}\right), 1713,1680(\mathrm{C}=\mathrm{O}), 1622(\mathrm{C}=\mathrm{C}), 1460(\mathrm{C}-\mathrm{N}) .{ }^{1} \mathrm{H}-\mathrm{NMR}$ $\left(500 \mathrm{MHz}, D M S O-d_{6}, p p m\right): \delta 13.43(1 \mathrm{H}, \mathrm{s}, \mathrm{NH}) ; 10.34(1 \mathrm{H}$, s, NH); $8.66(1 \mathrm{H}, \mathrm{s}, \mathrm{OH}) ; 8.06(1 \mathrm{H}, \mathrm{s}, \mathrm{H}-5$ '); $7.83(1 \mathrm{H}, \mathrm{s}$, H-4"); 7.21 (1H, s, H-6"); 6.99 (1H, d, $J=6.0 \mathrm{~Hz}, \mathrm{H}-7 ")$; 4.94 (2H, s, H-6'a, H-6'b); 4.29 (2H, s, H-5a, H-5b); 2.27 $\left(3 \mathrm{H}, \mathrm{s},-\mathrm{CH}_{3}\right) ; 1.95(2 \mathrm{H}, \mathrm{s}, \mathrm{H}-2 \mathrm{a}, \mathrm{H}-2 \mathrm{~b}) ; 1.74(2 \mathrm{H}, \mathrm{s}, \mathrm{H}-$ 4a, H-4b); 1.42 (2H, s, H-3a, H-3b). ${ }^{13} \mathrm{C} N M R(125 \mathrm{MHz}$, DMSO-d $\left.d_{6}, p p m\right): \delta 168.25,163.25,144.08,142.45,140.86$, $132.61,132.25,127.86,123.74,115.83,109.89,49.54,35.17$, $32.00,29.69,22.52,20.97$. HR-MS (ESI) $\mathrm{m} / \mathrm{z}$ calculated for $\mathrm{C}_{17} \mathrm{H}_{20} \mathrm{~N}_{6} \mathrm{O}_{4},[\mathrm{M}-\mathrm{H}]^{-}$371.1473. Found 371.1484.

2.1.2p N-Hydroxy-5-(4-((3-(hydroxyimino)-5-methoxy2-oxoindolin-1-yl)methyl)-1H-1,2,3-triazol-1-yl)pentanamide (6f): Yellow solid; Yield: 64\%. M.p.: 196.0-197.5 ${ }^{\circ} \mathrm{C} . R_{f}=0.48$ (DCM : $\left.\mathrm{MeOH}: \mathrm{AcOH}=90: 5: 1\right) . I R$ $\left(\mathrm{KBr}, \mathrm{Cm}^{-1}\right): 3421(\mathrm{NH}), 3134(\mathrm{OH}), 3047(\mathrm{C}-\mathrm{H}$, aren $), 2929$, $2838\left(\mathrm{CH}, \mathrm{CH}_{2}\right), 1718,1628(\mathrm{C}=\mathrm{O}), 1598(\mathrm{C}=\mathrm{C}), 1480(\mathrm{C}-$ N). ${ }^{1} H-N M R\left(500 M H z, D M S O-d_{6}, p p m\right): \delta 10.35(1 \mathrm{H}, \mathrm{s}$, $\mathrm{NH}) ; 8.67(1 \mathrm{H}, \mathrm{s}, \mathrm{OH}) ; 8.05(1 \mathrm{H}, \mathrm{s}, \mathrm{H}-5) ; 7.57(1 \mathrm{H}, \mathrm{d}, J$ $=2.5 \mathrm{~Hz}, \mathrm{H}-4$ "); 7.03-6.98 (2H, m, H-6", H-7"); 4.94 (2H, s, H-6'a, H-6'b); 4.29 (2H, t, $J=7.0 \mathrm{~Hz}, \mathrm{H}-5 \mathrm{a}, \mathrm{H}-5 \mathrm{~b}$ ); 3.72 $\left(3 \mathrm{H}, \mathrm{s},-\mathrm{OCH}_{3}\right) ; 1.95(2 \mathrm{H}, \mathrm{t}, J=7.0 \mathrm{~Hz}, \mathrm{H}-2 \mathrm{a}, \mathrm{H}-2 \mathrm{~b}) ; 1.75-$ 1.72 (2H, m, H-4a, H-4b); 1.45-1.40 (2H, m, H-3a, H-3b). ${ }^{13} C_{\text {NMR }}\left(125 \mathrm{MHz}, \mathrm{DMSO}-d_{6}\right.$, ppm): $\delta 168.74,162.66$, $155.25,143.71,141.87,136.25,123.24,116.90,115.87$, $113.14,110.28,55.68,49.07,34.73,31.54,29.22,22.06$. HRMS (ESI) $m / z$ calculated for $\mathrm{C}_{17} \mathrm{H}_{20} \mathrm{~N}_{6} \mathrm{O}_{5}[\mathrm{M}-\mathrm{H}]^{-} 387.1422$. Found 387.1440.

\subsection{2q5-(4-((7-Chloro-3-(hydroxyimino)-2-oxoindolin-} 1-yl)methyl)-1H-1,2,3-triazol-1-yl)-N-hydroxypentanamide (6g): Yellow solid; Yield: 73\%. M.p.: $198.5-201.0^{\circ} \mathrm{C}$. $R_{f}=0.47(\mathrm{DCM}: \mathrm{MeOH}: \mathrm{AcOH}=90: 5: 1) \cdot I R\left(\mathrm{KBr}, \mathrm{cm}^{-1}\right)$ : $3422(\mathrm{NH}), 3165(\mathrm{OH}), 3057(\mathrm{C}-\mathrm{H}$, aren), 2959, $2866(\mathrm{CH}$, $\left.\mathrm{CH}_{2}\right), 1724,1618(\mathrm{C}=\mathrm{O}), 1608(\mathrm{C}=\mathrm{C}), 1469(\mathrm{C}-\mathrm{N}) .{ }^{1} H-N M R$ $\left(500 \mathrm{MHz}, D M S O-d_{6}, p p m\right): \delta 13.83(1 \mathrm{H}, \mathrm{s}, \mathrm{NH}) ; 10.34(1 \mathrm{H}$, s, NH); $8.66(1 \mathrm{H}, \mathrm{s}, \mathrm{OH}) ; 8.07(1 \mathrm{H}, \mathrm{d}, J=7.0 \mathrm{~Hz}, \mathrm{H}-4$ "); 8.03 $(1 \mathrm{H}, \mathrm{s}, \mathrm{H}-5$ '); $7.39(1 \mathrm{H}, \mathrm{d}, J=8.0 \mathrm{~Hz}, \mathrm{H}-6$ "); $7.11(1 \mathrm{H}, \mathrm{t}, J$ $=7.5 \mathrm{~Hz}, \mathrm{H}-5$ "); 5.31 (2H, s, H-6'a, H-6'b); 4.29 (2H, t, $J=$ $7.0 \mathrm{~Hz}, \mathrm{H}-5 \mathrm{a}, \mathrm{H}-5 \mathrm{~b}) ; 1.95$ (2H, t, $J=7.25 \mathrm{~Hz}, \mathrm{H}-2 \mathrm{a}, \mathrm{H}-2 \mathrm{~b}$ ); 1.77-1.71 (2H, m, H-4a, H-4b); 145-1.39 (2H, m, H-3a, H3b). ${ }^{13}$ C NMR (125 MHz, DMSO- $\left.d_{6}, p p m\right): \delta 168.67,163.63$,
$143.26,142.20,138.45,133.77,125.84,124.22,122.44$, $118.29,114.80,49.01,37.06,31.50,29.24,22.01$. HR-MS (ESI) $m / z$ calculated for $\mathrm{C}_{16} \mathrm{H}_{17} \mathrm{ClN}_{6} \mathrm{O}_{4}[\mathrm{M}-\mathrm{H}]^{-} 391.0927$. Found 391.0918.

\subsection{Biological evaluation}

2.2a Cytotoxicity assay: The cytotoxicity of the synthesized compounds was evaluated against three human cancer cell lines, including SW620 (colon cancer), PC3 (prostate cancer), and AsPC-1 (pancreatic cancer). The cell lines were purchased from a Cancer Cell Bank at the Korea Research Institute of Bioscience and Biotechnology (KRIBB). The media, sera and other reagents that were used for cell culture in this assay were obtained from GIBCO Co. Ltd. (Grand Island, New York, USA).The cells were culture in DMEM (Dulbecco's Modified Eagle Medium) until confluence. The cells were then trypsinized and suspended at $3 \times 10^{4}$ cells $/ \mathrm{mL}$ of cell culture medium. On day 0 , each well of the 96-well plates was seeded with $180 \mu \mathrm{L}$ of cell suspension. The plates were then incubated in a $5 \% \mathrm{CO}_{2}$ incubator at $37{ }^{\circ} \mathrm{C}$ for 24 h. Compounds were initially dissolved in dimethyl sulfoxide (DMSO) and diluted to appropriate concentrations by culture medium. Then $20 \mu \mathrm{L}$ of each sample of compounds, which were prepared as described above, were added to each well of the 96-well plates, which had been seeded with cell suspension and incubated for $24 \mathrm{~h}$, at various concentrations. The plates were further incubated for $48 \mathrm{~h}$. Cytotoxicity of the compounds was measured by the colorimetric method, as described previously. ${ }^{21}$ with slight modifications. ${ }^{22-24}$ The $\mathrm{IC}_{50}$ values were calculated using a Probits method, ${ }^{25}$ taking average values from three independent determinations $(\mathrm{SD} \leq$ $10 \%)$.

2.2b HDAC2 enzyme assay: The HDAC2 enzyme was purchased from BPS Bioscience (San Diego, CA, USA). The HDAC enzymatic assay was performed using a Fluorogenic HDAC Assay Kit (BPS Bioscience) according to the manufacturer's instructions. Briefly, HDAC2 enzymes were incubated with vehicle or various concentrations of the assayed samples or SAHA for $30 \mathrm{~min}$ at $37^{\circ} \mathrm{C}$ in the presence of an HDAC fluorimetric substrate. The HDAC assay developer (which produces a fluorophore in reaction mixture) was added, and the fluorescence was measured using VICTOR ${ }^{3}$ (PerkinElmer, Waltham, MA, USA) with excitation at $360 \mathrm{~nm}$ and emission at $460 \mathrm{~nm}$. The measured activities were subtracted by the vehicle-treated control enzyme activities and $\mathrm{IC}_{50}$ values were calculated using GraphPad Prism (GraphPad Software, San Diego, CA, USA).

\subsection{Docking studies}

An AutoDockVina program (The Scripps Research Institute, CA, USA)was used for docking. ${ }^{26}$ The structure of HDAC2 protein in complex with SAHA was obtained from the Protein Data Bank (PDB) (PDB ID: 4LXZ). ${ }^{27}$ The 
coordinates of the compounds were generated by using the GlycoBioChem PRODRG2 Server (http://davapc1.bioch.dun dee.ac.uk/prodrg/). ${ }^{28}$ For the docking studies, the grid maps were centered on the SAHA binding site and comprised $26 \times 26 \times 22$ points with $1.0 \AA$ spacing after SAHA was removed from the complex structure, as described in previous works. ${ }^{17,18,20}$ The AutoDockVina program was performed using eight-way multithreading and the other parameters were default settings.

\subsection{Prediction of absorption, distribution, metabolism, elimination, and toxicity (ADMET)}

In this work ADMET-related properties were computed using Volsurf+ v.1.0.4 and the Chemistry Development Kit (CDK) software (http://cdk.sourceforge.net/). Compound topology was saved as SMILES format (simplified molecular-input line-entry system) using MarvinSketch and further submitted to Volsurf and CDK for molecular descriptor computation. ADMET descriptors comprise Blood-Brain Barrier distribution $(\log \mathrm{BB})$, percentage of protein binding $(\mathrm{PB}, \%)$, volume of distribution (VD, $\mathrm{L} / \mathrm{kg}$ ) and metabolic stability against human CYP3A4 enzyme (MetStab, \%). The ability to interact with cytochrome enzyme isoforms (substrate and/or inhibition of CYP3A4, 1A2, 2C9, 2C19, and 2D6) was also checked using admetSAR server developed by Cheng et al. ${ }^{29}$ Physicochemical descriptors including molecular weight (MW), partition coefficients ( $\left.\log \mathrm{P}, \operatorname{clog} \mathrm{D}_{\mathrm{pH}=7.4}\right)$, total polar surface area (PSA, $\AA^{2}$ ), number of hydrogen bond donors and acceptors, thermodynamic and $\mathrm{pH}$-dependent aqueous solubility $(\mathrm{mg} / \mathrm{ml})$ were calculated. In addition, 3PRule was applied to classify permeability across adenocarcinoma (Caco-2) cell membrane ${ }^{30}$ - a model commonly used for intestinal absorption estimation. The P-glycoprotein (P-gp, MDR1) substrate states were identified using chemoinformatic approaches developed by Levatic et al. ${ }^{31}$ (available at http://pgp.biozyne. com). Lipinski's Rule of Five (Ro5) was also applied to reveal drug-likeness of designed compounds. ${ }^{32}$ Finally, in vivo toxicity was predicted by analyzing two risk factors (clogP and PSA) following the same criteria described by Hughes et al. ${ }^{33}$

\section{Results and Discussion}

\subsection{Chemistry}

The targeted hydroxamic acids 4-6 were synthesized via three-step pathway, as illustrated in Scheme 1. The first step was a nucleophilic substitution between isatins $\mathbf{1}$ and propargyl bromide under basic conditions $\left(\mathrm{K}_{2} \mathrm{CO}_{3}\right)$ in dimethylformamide (DMF) with a catalytic amount of KI to furnish 1-propargylisatins 2 . In the second step, a Click reaction between propargyl compounds 2 and respective methyl azidoalkanoates (including methyl 3-azidopropanoate, methyl 4-azidobutanoate, methyl 5-azidovalerate) gave the intermediate esters 3 .
This reaction proceeded smoothly in acetonitrile as a solvent and copper iodide as a catalyst. In most cases, the ester intermediates were precipitated by titration of the reaction residues, which were obtained by evaporation of acetonitrile, with cold water. The final step in the pathway involved a nucleophilic acyl substitution of hydroxylamine hydrochloride with the esters $\mathbf{3}$. This reaction occurred under alkaline conditions in a solvent mixture which included tetrahydrofuran and methanol at $0-5{ }^{\circ} \mathrm{C}$. The overall yields of compounds 4-6 were moderate.

The structures of the synthesized compounds were determined straightforwardly based on analysis of spectroscopic data, including IR, MS, ${ }^{1} \mathrm{H}$ and ${ }^{13} \mathrm{C}$ NMR (Supporting Information). The concurrent condensation of the hydroxylamine with the oxo group at position 3 on the indoline ring under the above conditions occurred in all cases. This condensation has been observed and fully explained previously. ${ }^{19}$

\subsection{Bioactivity}

Due to limited funding resource, in this investigation the compounds synthesized were preliminarily evaluated for histone deacetylase inhibition using HDAC2. We chose HDAC2 because it has been shown that deacetylation of a number of key histone proteins is regulated principally by HDAC2 and HDAC 3 . HDAC2 has been demonstrated to play very important role in promoting cellular proliferation, while inhibiting normal cell apoptosis. ${ }^{34}$ In addition, the compounds were also evaluated for cytotoxicity against three human cancer cell lines, including SW620 (colon cancer), PC3 (prostate cancer), and AsPC-1 (pancreatic cancer). The results are presented in Table 1.

When designing these compounds, we initially envisioned that the triazole moiety would add more benefits in hydrogen bonding with the amino acid regions in the active binding sites of HDAC, while still serving as a part of a linker between the 2-oxoindoline and hydroxamic acid moieties. Due to shorter length of the $\mathrm{C}=\mathrm{N}$ and $\mathrm{C}-\mathrm{N}$ bonds compared to $\mathrm{C}-\mathrm{C}$ bond, we estimated that if there were only $2 \mathrm{C}$ between the triazole and hydroxamic acids, the linker between the 2-oxoindoline and hydroxamic acid moieties (in compounds 4a-c) would be shorter than 6C linker in SAHA. Therefore we decided to design series 5a-g with $\mathrm{n}=$ 1 as the target compounds. Series 6a-g with one extra $\mathrm{C}$ was also synthesized to compare with series 5a-g. The results presented in Table 1 demonstrate that in series 5a-g and 6a-g, with the exception of a 5-fluorine substituent, other substituents at either position 5 or 7 
Table 1. Inhibition of HDAC activity and cytotoxicity of the compounds synthesized against several human cancer cell lines.

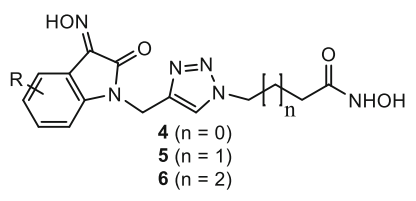

\begin{tabular}{|c|c|c|c|c|c|c|c|}
\hline \multirow[t]{2}{*}{ Cpd code } & \multirow[t]{2}{*}{$\mathrm{R}$} & \multirow[t]{2}{*}{$\mathrm{n}$} & \multirow[t]{2}{*}{$\log P^{1}$} & \multirow[t]{2}{*}{ HDAC2 inhibition $\left(\mathrm{IC}_{50},{ }^{2} \mu \mathrm{M}\right)$} & \multicolumn{3}{|c|}{ Cytotoxicity $\left(\mathrm{IC}_{50},{ }^{2} \mu \mathrm{M}\right) /$ Cell lines } \\
\hline & & & & & SW620 & PC3 & AsPC-1 \\
\hline $4 a$ & $\mathrm{H}$ & 0 & 0.41 & 1.70 & 29.0 & $>30$ & $>30$ \\
\hline $4 b$ & $5-\mathrm{F}$ & 0 & 0.61 & 6.24 & $>30$ & $>30$ & $>30$ \\
\hline $4 c$ & $5-\mathrm{Cl}$ & 0 & 1.05 & 2.80 & $>30$ & $>30$ & $>30$ \\
\hline $5 \mathbf{a}$ & $\mathrm{H}$ & 1 & 0.90 & 6.16 & 26.26 & $>30$ & 26.87 \\
\hline $5 b$ & $5-\mathrm{F}$ & 1 & 1.10 & 8.27 & 23.64 & $>30$ & $>30$ \\
\hline $5 c$ & $5-\mathrm{Cl}$ & 1 & 1.54 & 1.72 & 13.46 & 16.28 & 11.60 \\
\hline $5 d$ & $5-\mathrm{Br}$ & 1 & 1.79 & 3.53 & 2.93 & 6.08 & 3.01 \\
\hline $5 e$ & $5-\mathrm{CH}_{3}$ & 1 & 1.44 & 1.28 & 0.73 & 0.76 & 0.49 \\
\hline $5 f$ & $5-\mathrm{OCH}_{3}$ & 1 & 0.98 & 0.91 & 1.61 & 1.74 & 1.49 \\
\hline $5 g$ & 7-Cl & 1 & 1.54 & 5.08 & 10.58 & 9.27 & 12.90 \\
\hline $6 a$ & $-\mathrm{H}$ & 2 & 1.39 & 4.87 & $>30$ & $>30$ & $>30$ \\
\hline $6 b$ & $5-\mathrm{F}$ & 2 & 1.59 & 26.64 & $>30$ & $>30$ & $>30$ \\
\hline $6 c$ & $5-\mathrm{Cl}$ & 2 & 2.03 & 2.65 & 9.16 & 4.69 & 4.51 \\
\hline $6 d$ & $5-\mathrm{Br}$ & 2 & 2.28 & 2.16 & 5.64 & 3.42 & 4.43 \\
\hline $6 e$ & $5-\mathrm{CH}_{3}$ & 2 & 1.94 & 3.52 & $>30$ & $>30$ & $>30$ \\
\hline $6 f$ & $5-\mathrm{OCH}_{3}$ & 2 & 1.47 & 4.15 & $>30$ & $>30$ & $>30$ \\
\hline $6 \mathrm{~g}$ & 7-Cl & 2 & 2.03 & 4.77 & $>30$ & $>30$ & $>30$ \\
\hline SAHA $^{4}$ & & & 1.44 & 1.06 & 3.20 & 3.70 & 3.75 \\
\hline
\end{tabular}

${ }^{1}$ Calculated by ChemDraw 9.0 software; ${ }^{2}$ The concentration $(\mu \mathrm{M})$ of compounds that produces a $50 \%$ reduction in enzyme activity or cell growth, the numbers represent the averaged results from triplicate experiments with deviation of less than 10\%.; ${ }^{3}$ Cell lines: SW620, colon cancer; PC3, prostate cancer; AsPC-1, pancreatic cancer; ${ }^{4}$ SAHA, suberoylanilide acid, a positive control.

on the 2-oxoindoline ring generally enhanced HDAC2 inhibition. Also, in overall, series 5a-g inhibited the activity of HDAC2 more potently compared to series 6a-g. A similar trend was also observed with cytotoxicity when comparing series 5a-g and series 6a-g. In series $\mathbf{5 a - g}$, it was found that the relationships between HDAC2 inhibition and cytotoxicity of the compounds were relatively well correlated. Compounds $\mathbf{5 d}$ and $\mathbf{5 e}$, which were the most potent HDAC2 inhibitors in the series, were also found to be the most cytotoxic against all three cancer cell lines tested. In contrast, compounds $\mathbf{5 b}$ and5g, which were the least potent HDAC2 inhibitors in the series, were also among the least cytotoxic ones. From the results obtained, it is suggested that, when considering the 4-methyl-1 $H$-1,2,3-triazole moiety as a part of a linker between the 3-oxoindoline system and hydroxamic acid group, an alkyl length of three carbon connecting hydroxamic acid and triazole $1 \mathrm{H}$ 1,2,3-triazole moieties would be most favorable for bioactivities.

Among the compounds synthesized, compound 5f was found to exhibit similar potency in term of HDAC2 inhibition compared to SAHA, meanwhile compound $\mathbf{5 e}$ emerged as the most potential candidate with cytotoxicity or up to 8-fold more potent than SAHA in all three cancer cell lines tested. It could be seen that in these two compounds the correlation between HDAC2 inhibitory potency and cytotoxicity was not finely observed. Some reasons might explain for this discrepancy. First, compound 5e could be more potently inhibit other types of HDACs of class I and class II, which are also important in promoting cell proliferation and inhibiting cellular apoptosis. Second, from $\log \mathrm{P}$ values, it would be possible that compound $\mathbf{5 f}$ (with lower $\log \mathrm{P}$ value of 0.98 ) was not as good as compound $\mathbf{5 e}$ (with higher $\log \mathrm{P}$ value of 1.44, similar to that of SAHA) in penetrating through cellular membrane.

\subsection{Docking studies and structure-activity relationships}

It has been shown that histone-H3 and histone-H4 deacetylation is regulated principally by HDAC2 and 
HDAC3. ${ }^{34}$ and the crystal structure of HDAC2 in complex with SAHA (PDB ID: 4LXZ) has been reported by Lauffer and co-workers. ${ }^{27}$ So we decided to select the structure of SAHA-HDAC2 complex a template in docking experiments to study the structureactivity relationships of these hydroxamic acids and HDAC. Firstly, a control re-docking with co-crystal SAHA to the crystal structures of HDAC2 was executed following the procedures reported previously. ${ }^{17,19,20}$ The results showed very similar interaction pattern between re-docked and the original SAHA in the crystal structure (the all-atom root-mean-square deviation of 0.609 $\AA$ ). It was found from docking experiments that all of the hydroxamic acids synthesized were well located in the active site of the enzyme with binding affinities $(\Delta \mathrm{G})$ between -6.7 and $-8.1 \mathrm{kcal} / \mathrm{mol}$, comparable or lower than that of SAHA (Table 2). It is noted that, in some instances the predicted binding affinities of the compounds were not readily correlated to the experimental data obtained from HDAC2 inhibition assay. For example, the stabilization energies of predicted binding modes on HDAC2 of compounds $\mathbf{5 b}$ and $\mathbf{5} \mathbf{c}$ were found to be quite similar $(-7.7$ and $-7.8 \mathrm{kcal} / \mathrm{mol}$, respectively). These results were not explainable for the 5-fold difference in the HDAC2 inhibitory effects of compounds $\mathbf{5 b}$ and $\mathbf{5 c}$. Especially in case of compound $\mathbf{5 f}$, which has the same calculated stabilization energies as SAHA $(-7.4 \mathrm{kcal} / \mathrm{mol})$ was found to exhibit similar potency compared to SAHA in HDAC2 inhibition $\left(\mathrm{IC}_{50}\right.$ value was $0.91 \mu \mathrm{M}$ vs. $1.06 \mu \mathrm{M}$ of SAHA). Compounds $5 \mathbf{e}(\Delta \mathrm{G}=-8.1 \mathrm{kcal} / \mathrm{mol})$ and $5 \mathbf{f}$ were two most potent in the series in term of HDAC2 inhibition. Interestingly however, the length of aliphatic chain linking hydroxamic group with triazole ring appeared to be an important factor. There was a remarkable drop in the docking affinity ranges from butanamide to pentanamide moieties, which suggested the negative effect of lengthening the aliphatic chain beyond fourcarbons.

From the docking experiments, it was also found that a zinc ion (grey sphere), which was coordinated by three residues of HDAC2, including Asp181, His183 and Asp269, interacted with all the hydroxamic acid groups in a similar manner as SAHA did. This zinc-chelating functionality has been identified as the dominant factor for inhibitor potency of synthesized hydroxamic derivatives. For all the compounds, it was found that a 1-alkyl-4-methyl-1 $H$-1,2,3-triazole linker between the indoline and hydroxamic acid moieties was tightly stacked between Phe155 and Phe210 residues of the enzymes (Figures 3,4) and this pi-stacking interaction could be the key factor attributing to the good binding affinities of the compounds with HDAC2.
Interestingly, methyl and methoxy substituents enhanced the interactions between oxoindoline groups and hydrophobic residues at the rim of the pocket, such as Pro34 and Leu276. In these docking experiments, however, the indoline part was found to insignificantly interact with the enzyme. Subsequently, very little variance in the binding affinities among the compounds with different substituted groups was observed. The most potent HDAC2 inhibitor compound $\mathbf{5 e}$ and $\mathbf{5 f}$, exhibited extensive hydrogen bonds with His146, Asp181, Glu208 and Tyr308.

\subsection{ADMET prediction}

Today, it is of great interest for modern medicinal chemistry to use computational tools to predict the ADMET properties in parallel with optimizing the bioactive potency of novel chemical entities. ${ }^{35}$ Based on the cytotoxicity as well as HDAC2 inhibition potency, we decided to further investigate the ADMET profiles of six derivatives (5a-f and $\mathbf{6 c - d}$ ) from synthesized compounds using computational approaches.

Firstly, accomplishment of Lipinski's Ro5 was checked in order to roundly predict the drug-likeness of chemicals. ${ }^{32}$ All the compounds exceeded the number of five hydrogen bond acceptors, and predicted as possible non-oral drug candidates since recent studies have suggested extending the chemical space beyond Ro5 to avoid lost opportunities, especially for new anticancer agents. ${ }^{36}$

As can be appreciated from Table 3, there is no significant difference of ADME properties calculated. Considering the chemicals as drug-like candidates, we are now discussing some details about the predicted values. Compounds $\mathbf{5 e}$ and $\mathbf{5 f}$ are very slightly soluble in water (solubility $\geq 0.01 \mathrm{mg} / \mathrm{mL}$ ) while others are identified as insoluble ones. ${ }^{37}$ Additionally, based on 3PRule developed by Pham-The et al. ${ }^{30}$ all the compounds were classified as low intestinal permeability. As stated previously, low permeable compounds are generally absorbed via gastrointestinal tract in a wide range of extent. ${ }^{37}$ Interestingly, the poor relationship between permeability classes and lipophilicity $(\log \mathrm{P})$ suggests that the transport mechanisms of these analogues are others than passive diffusion. ${ }^{30}$ Other ADME parameters of interest were those related to the first-pass metabolism such as P-gp substrate and metabolic stability against cytochrome P450 enzymes. Accordingly, all the compounds were identified to be highly resistant to both P-gp and CYP3A4 metabolism factors. A further inspection of metabolism interactions towards different CYP isoforms revealed that all compounds are 
Table 2. Binding affinities of compounds 4-6 towards HDAC2.

\begin{tabular}{|c|c|c|c|c|c|c|c|c|}
\hline Cpd. code & $\begin{array}{l}\text { Binding } \\
\text { Affinity } \\
\text { (kcal/mol) }\end{array}$ & $\begin{array}{l}\text { HDAC2 } \\
\text { inhibition } \\
\left(\mathrm{IC}_{50}, \mu \mathrm{M}\right)\end{array}$ & Cpd. code & $\begin{array}{l}\text { Binding } \\
\text { Affinity } \\
\text { (kcal/mol) }\end{array}$ & $\begin{array}{l}\text { HDAC2 } \\
\text { inhibition } \\
\left(\mathrm{IC}_{50}, \mu \mathrm{M}\right)\end{array}$ & Cpd. code & $\begin{array}{l}\text { Binding } \\
\text { Affinity } \\
\text { (kcal/mol) }\end{array}$ & $\begin{array}{l}\text { HDAC2 } \\
\text { inhibition } \\
\left(\mathrm{IC}_{50}, \mu \mathrm{M}\right)\end{array}$ \\
\hline $4 \mathbf{a}$ & -7.4 & 1.70 & $5 d$ & -7.8 & 3.53 & $6 c$ & -7.1 & 2.65 \\
\hline $4 b$ & -7.6 & 6.24 & $5 e$ & -8.1 & 1.28 & 6d & -7.0 & 2.16 \\
\hline $4 c$ & -7.7 & 2.80 & $5 f$ & -7.4 & 0.91 & $6 e$ & -7.4 & 3.52 \\
\hline $5 \mathbf{a}$ & -7.5 & 6.16 & $5 g$ & -7.9 & 5.08 & $6 f$ & -7.4 & 4.15 \\
\hline $5 b$ & -7.7 & 8.27 & $6 \mathbf{a}$ & -6.7 & 4.87 & 6g & -7.2 & 4.77 \\
\hline $5 c$ & -7.8 & 1.72 & $6 \mathbf{b}$ & -7.0 & 26.64 & SAHA & -7.4 & 1.06 \\
\hline
\end{tabular}

Table 3. Physicochemical and pharmacokinetic profiles of the synthesized compounds.

\begin{tabular}{lcccccccc}
\hline Cpd. Ro5* & $\begin{array}{l}\text { Solubility } \\
(\mathrm{mg} / \mathrm{ml})\end{array}$ & $\begin{array}{l}\text { Caco-2 per- } \\
\text { meability } \\
\text { class }^{2}\end{array}$ & $\begin{array}{l}\text { BBB } \\
\text { distribution }\end{array}$ & P-gp substrate & $\begin{array}{l}\text { Metabolism } \\
\text { stability } \\
(\%)\end{array}$ & $\begin{array}{l}\text { Plasma } \\
\text { protein } \\
\text { binding }(\%)\end{array}$ & $\begin{array}{l}\text { Volume of } \\
\text { distribution } \\
(1 / \mathrm{kg})\end{array}$ \\
\hline $\mathbf{5 c}$ & 1 & $5.62 \times 10^{-3}$ & Low & Very low & No & 100.00 & 51.66 & 0.68 \\
$\mathbf{5 d}$ & 1 & $2.88 \times 10^{-3}$ & Low & Very low & No & 100.00 & 55.86 & 0.74 \\
$\mathbf{5 e}$ & 1 & $9.12 \times 10^{-3}$ & Low & Very low & No & 100.00 & 44.10 & 0.67 \\
$\mathbf{5 f}$ & 1 & $2.95 \times 10^{-2}$ & Low & Very low & No & 100.00 & 39.62 & 0.57 \\
$\mathbf{6 c}$ & 1 & $3.02 \times 10^{-3}$ & Low & Very low & No & 93.78 & 55.27 & 0.82 \\
6d & 1 & $1.58 \times 10^{-3}$ & Low & Very low & No & 88.29 & 59.42 & 0.85 \\
\hline
\end{tabular}

*Number of Lipinski Rule of five violations; ${ }^{1}$ intrinsic solubility at $25{ }^{\circ} \mathrm{C}$ calculated by Volsurf +1.0 .4 (VolSurf + , version 1.0.4 edn. available from Molecular Discovery Ltd., London, U.K. (http://www.moldiscovery.com)); ${ }^{2}$ Caco-2 cell permeability classification using 3PRule: ${ }^{30}$ High class if $\mathrm{P}_{a p p} \geq 16 \times 10^{6} \mathrm{~cm} / \mathrm{s}$, Moderate class if $0.7 \times 10^{-6} \leq$ $\mathrm{P}_{\text {app }}<16 \times 10^{-6} \mathrm{~cm} / \mathrm{s} ;{ }^{3}$ Blood-Brain Barrier distribution classes based on Volsurf+1.0.4 logBB descriptor: Moderate class if $0 \leq \log \mathrm{BB}<0.5$, Low class if $-0.3 \leq \log \mathrm{BB}<0$, and Very low class if $\log \mathrm{BB}<-0.3 ;{ }^{4} \mathrm{P}$-glycoprotein efflux substrate state identified via online server http://pgp.biozyne.com; ${ }^{5}$ Metabolic stability in human CYP3A4 enzyme estimated by Volsurf+1.0.4: a value greater than $50 \%$ corresponds to metabolic stable compound.

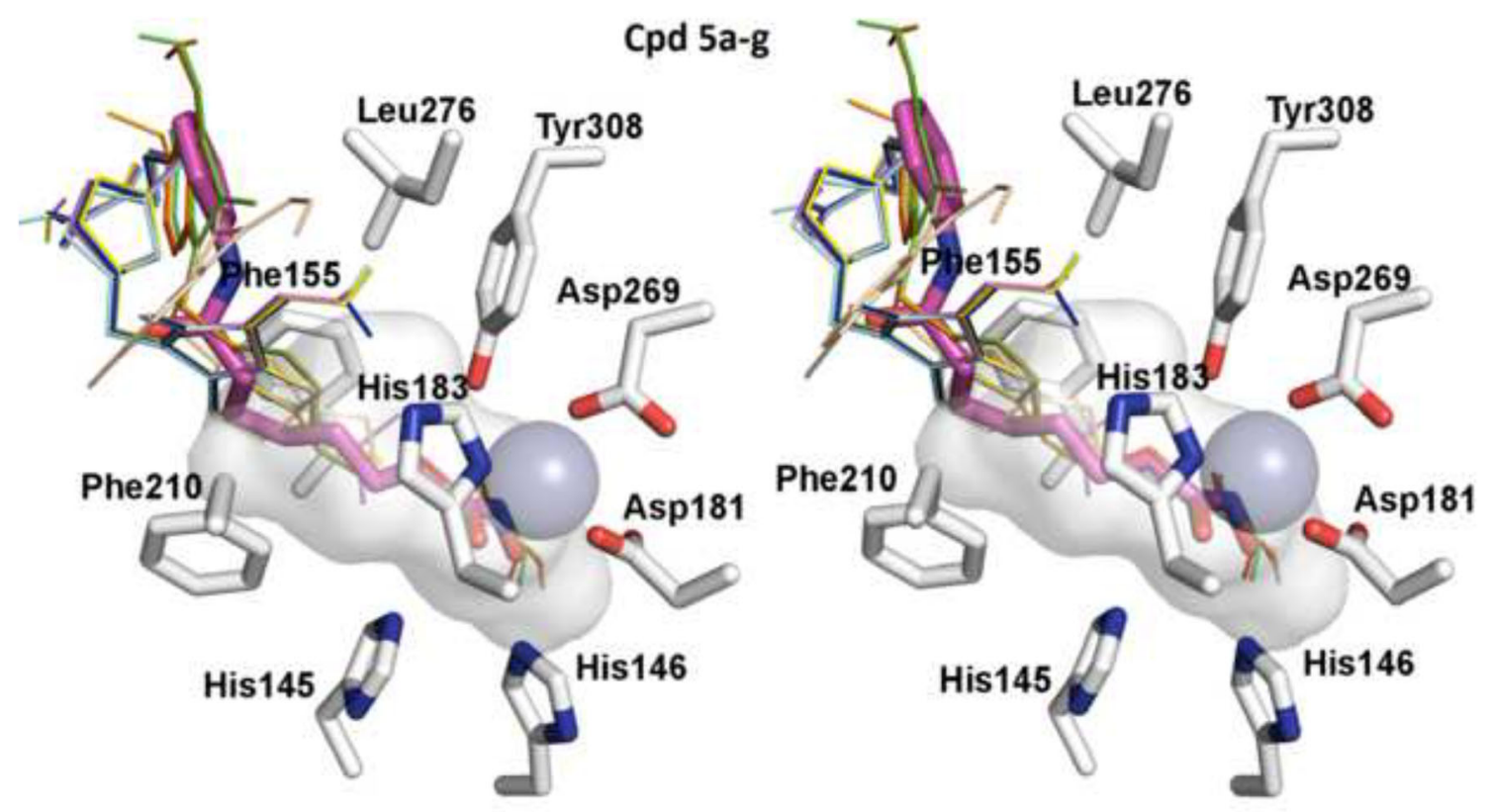

Figure 3. Stereo-view of the overlapping of the compounds 5a-g and SAHA's binding modes at the binding site of HDAC2. Compounds are represented as a stick model. SAHA presented as bold magenta stick. 


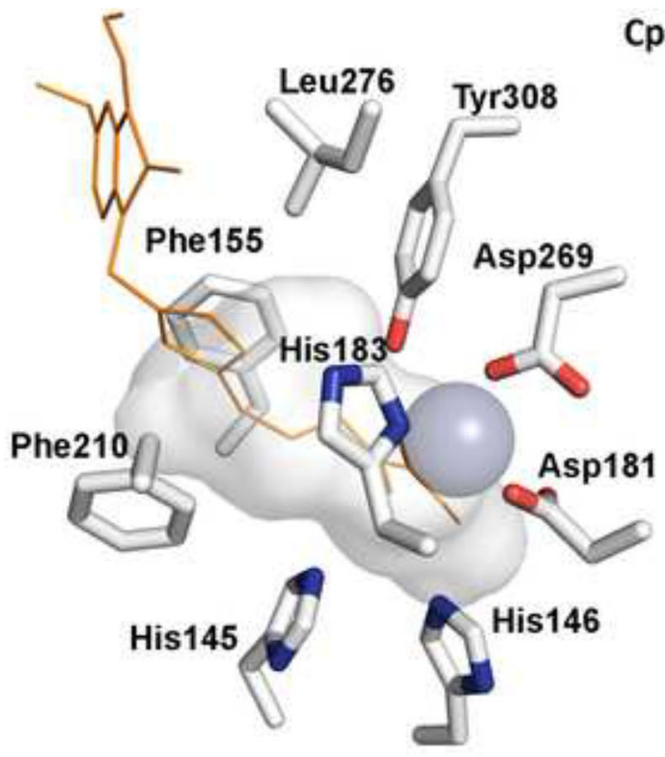

Cpd $5 f$

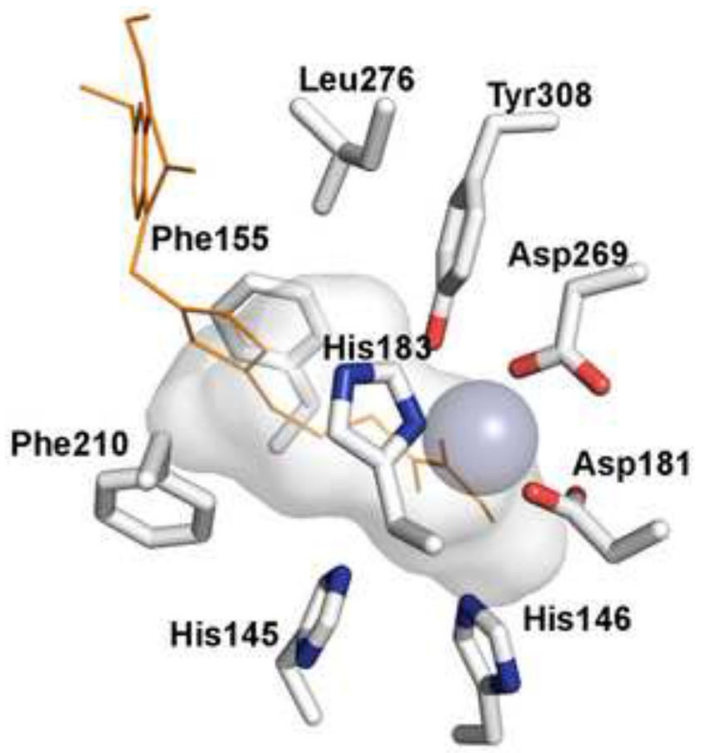

Figure 4. Stereo-view presentations of the actual binding poses of SAHA and simulated docking poses of compound $\mathbf{5 f}$ to HDAC2. A ligand is represented as a stick model shown in dark-orange and yellow-orange color, respectively. The most important parts for the enzyme for interaction of these compounds were shown as a stick model with carbon, nitrogen, and oxygen colored as grey, blue and red, respectively. $\mathrm{Zn}^{2+}$ ion is shown as a bright gray sphere.

both substrate and inhibitor of CYP3A4. They were also predicted to be inhibitors of CYP2C9 while did not interact with other isoforms. On the other hand, the information of plasma protein (mainly albumin) binding (PB) and volume of distribution (VD) is helpful for establishing safety margins and dose regimen of drug candidates. ${ }^{38}$ In this aspect, compounds $\mathbf{5 e}$ and $\mathbf{5 f}$ (with the lowest PB value) could be considered safer than the others. With respect to the drug distribution parameter, all analogues could be moderately distributed in body tissue rather than the plasma since $\mathrm{VD}>0.57 \mathrm{l} / \mathrm{kg}$, which is estimated to be total body water volume (> $60 \%$ body weight). ${ }^{39}$ They also displayed a very low disposition profile in the central nervous system. ${ }^{40}$

Lastly, in vivo toxicity of all the compounds was predicted based on two risk factor approaches developed by Hughes and colleagues. ${ }^{33}$ The results indicated that the calculated properties are within the drug-like range and any compound concurrently presented both risk factors, such as $\log \mathrm{P}>3$ and PSA $<75 \AA^{2}$. Especially, 5e and $\mathbf{5 f}$ have desirable characteristics for anticancer drug candidate.

\section{Conclusions}

In conclusion, we have reported a series of 3hydroxyimino-2-oxoindoline-based hydroxamic acids incorporated with 1-alkyl-4-methyl-1 $H$-1,2,3-triazole linker showing strong HDAC2 inhibitory effects and potent cytotoxicity against several human cancer cell lines, including SW620 (human colon cancer), PC3 (prostate cancer) and AsPC-1 (pancreas cancer). The results we obtained from this study again confirm that 3-hydroxyimino-2-oxoindolines could well serve as a cap group for hydroxamic acid HDAC inhibitors. Also, different substituents on the benzene ring of the 3-hydroxyimino-2-oxoindoline moiety substantially influence both HDAC inhibition and cytotoxicity of the resulting compounds. Especially, 1-alkyl-4-methyl-1 $H$-1,2,3-triazole linker is tolerable for HDAC inhibitory activity. Computational predictions suggested that compounds $\mathbf{5 e}$ and $\mathbf{5 f}$, while showing potent HDAC2 inhibitory bioactivity, hold desirable ADMET characteristics for anticancer compounds. From this study, more extensive bioevaluation and ADMET study are being continued to investigate the potentials of these compounds as anticancer agents.

\section{Supplementary information (SI)}

All ${ }^{1} \mathrm{H}$ NMR, ${ }^{13} \mathrm{C}$ NMR and MS spectra can be found in Supplementary Information which is available free of charge at www.ias.ac.in/chemsci.

\section{Acknowledgements}

We acknowledge the principal financial supports from the National Foundation for Science and Technology of Vietnam 
(NAFOSTED, Grant number 104.01-2017.301). The work was also partly supported by the project No. 2017R1A5A201 5541 (from NRF, Korea, for extra-biology evaluation) and project No. QG.16.14 (from VNU for L-T-T. Huong to perform ADMET prediction which was not supported by NAFOSTED grant).

\section{Competing interests}

The authors report no conflict of interest.

\section{Authors' contributions}

N-H.N proposed the work. N-H.N, DTMD, DTA, VTM Hue mainly developed the synthesis studies. S-B.H, EJP, YJC and JSK performed the bioactivity testing assays. P-T.H, L-T-T.H, NTKY, BWH performed the docking simulations, ADMET and physicochemical computations. All authors have read and approved the final manuscript.

\section{References}

1. Nam N H and Parang K 2003 Current targets for anticancer drug discovery Curr. Drug Targets 4 159

2. Witt O, Deubzer H E, Milde T and Oehme I 2009 HDAC family: What are the cancer relevant targets? Cancer Lett. 2778

3. Ruijter A J M, Gennip A H, Caron H N, Kemp S and Kuilenburg A B P 2003 Histone deacetylases (HDACs): Characterization of the classical HDAC family Biochem. J. 370737

4. Zwergel C, Valente S, Jacob C and Mai A 2015 Emerging approaches for histone deacetylase inhibitor drug discovery Expert Opin. Drug Discov. 10599

5. West A C and Johnstone R W 2014 New and emerging HDAC inhibitors for cancer treatment J. Clin. Invest. 124 30

6. Hamm C A and Costa F F 2015 Epigenomes as therapeutic targets Pharmacol. Ther. 15172

7. Bolden J E, Peart M J and Johnstone R W 2006 Anticancer activities of histone deacetylase inhibitors Nat. Rev. Drug Discov. 5769

8. Dallavalle S, Cincinelli R, Nannei R, Merlini L, Morini G, Penco S, Pisano C, Vesci L, Barbarino M, Zuco V, De Cesare M and Zunino F 2009 Design, synthesis, and evaluation of biphenyl-4-yl-acrylohydroxamic acid derivatives as histone deacetylase (HDAC) inhibitors Eur. J. Med. Chem. 441900

9. Bracker T U, Sommer A, Fichtner I, Faus H, Haendler B and Han H-S 2009 Efficacy of MS-275, a selective inhibitor of class I histone deacetylases, in human colon cancer models Int. J. Oncol. 35909

10. Iyer S P and Foss F F 2015 Romidepsin for the Treatment of Peripheral T-Cell Lymphoma Oncologist 20 1084

11. Valente $\mathrm{S}$ and Mai A 2014 Small-molecule inhibitors of histone deacetylase for the treatment of cancer and noncancer diseases: A patent review (2011-2013) Expert. Opin. Ther. Pat. 24401
12. Jiyang L, Guangqiang L and Wenqing X 2013 Histone deacetylase inhibitors: An attractive strategy for cancer therapy Curr. Med. Chem. 201858

13. Ververis K, Hiong A, Karagiannis T C, Licciardi P V 2013 Histone deacetylase inhibitors (HDACIs): Multitargeted anticancer agents Biologics 747

14. Qiu T, Zhou L, Zhu W, Wang T, Wang J, Shu Y and Liu P 2013 Effects of treatment with histone deacetylase inhibitors in solid tumors: A review based on 30 clinical trials Future Oncol. 9255

15. Raedler L A 2016 Farydak (Panobinostat): First HDAC inhibitor approved for patients with relapsed multiple myeloma Am. Health Drug Benefits 984

16. Guha M 2015 HDAC inhibitors still need a home run, despite recent approval Nat. Rev. Drug Discov. 14365

17. Oanh D T K, Hai H V, Park S H, Kim H-J, Han B-W, Kim H-S, Hong J-T, Han S-B, Hue V T M and Nam N-H 2011 Benzothiazole-containing hydroxamic acids as histone deacetylase inhibitors and antitumor agents Bioorg. Med. Chem. Lett. 217509

18. Tung T, Kim O D T, Phuong D P T, Hue V T, Park S H, Han S W, Kim Y, Hong JT, Han S B and Nam N H 2013 New benzothiazole/thiazole-containing hydroxamic acids as potent histone deacetylase inhibitors and antitumor agents Med. Chem. 91051

19. Nam N-H, Huong T L, Mai Dung D T, Phuong Dung P T, Kim Oanh DT, Quyen D, Thao L T, Park S H, Kim K R, Han B W, Yun J, Kang J S, Kim Y and Han SB 2013 Novel isatin-based hydroxamic acids as histone deacetylase inhibitors and antitumor agents Eur. J. Med. Chem. 70477

20. Nam N H, Huong T L, Dung D T M, Dung P T P, Oanh D T K, Park S H, Kim K, Han S W, Yoon J E, Kang J S, Kim Y S and Han S B 2014 Synthesis, bioevaluation and docking study of 5-substitutedphenyl-1,3,4-thiadiazolebased hydroxamic acids as histone deacetylase inhibitors and antitumor agents J. Enzyme Inhib. Med. Chem. 29 611

21. Skehan P, Storeng R, Scudiero D, Monks A, Mc Mahon J, Vistica D, Warren J T, Bokesch H, Kenney S and Boyd M R 1990 New colorimetric cytotoxicity assay for anticancer-drug screening J. Natl. Cancer Inst. 821107

22. Thuong $\mathrm{P}$ T, Na M K, Dang N H, Hung T M, Ky P T, Thanh T V, Nam N H, Thuan N D, Sok D E and Bae K H 2006 Antioxidant activities of Vietnamese medicinal plants Nat. Prod. Sci. 1229

23. Ye G, Nam N-H, Kumar A, Saleh A, Shenoy D B, Amiji M M, Lin X, Sun G and Parang K 2007 Synthesis and evaluation of tripodal peptide analogues for cellular delivery of phosphopeptides J. Med. Chem. 503604

24. You Y-J, Kim Y, Nam N-H and Ahn B-Z 2003 Antitumor activity of unsaturated fatty acid esters of 4/-demethyldeoxypodophyllotoxin Bioorg. Med. Chem. Lett. 132629

25. Wu L, Smythe A M, Stinson S F, Mullendore L A, Monks A, Scudiero D A, Paull K D, Koutsoukos A D, Rubinstein L V, Boyd M R and Shoemaker R H 1992 Multidrugresistant phenotype of disease-oriented panels of human tumor cell lines used for anticancer drug screening Cancer Res. 523029

26. Trott O and Olson A J 2010 AutoDock Vina: Improving the speed and accuracy of docking with a new scoring 
function, efficient optimization, and multithreading $J$. Comput. Chem. 31455

27. Lauffer B EL, Mintzer R, Fong R, Mukund S, Tam C, Zilberleyb I, Flicke B, Ritscher A, Fedorowicz G, Vallero R, Ortwine D F, Gunzner J, Modrusan Z, Neumann L, Koth C M, Lupardus P J, Kaminker J S, Heise C E and Steiner P 2013 Histone Deacetylase (HDAC) inhibitor kinetic rate constants correlate with cellular histone acetylation but not transcription and cell viability J. Biol. Chem. 288 26926

28. Schuttelkopf A W and van Aalten D M F 2004 PRODRG: A tool for high-throughput crystallography of proteinligand Complexes Acta Cryst. D 601355

29. Cheng F, Li W, Zhou Y, Shen J, Wu Z, Liu G, Lee P W and Tang Y 2012 admetSAR: A comprehensive source and free tool for assessment of chemical ADMET properties J. Chem. Inf. Model 523099

30. Pham-The H, González-Álvarez I, Bermejo M, Garrigues T, Le-Thi-Thu H, Cabrera-Pérez M Á 2013 The use of rule-based and QSPR approaches in ADME profiling: A case study on Caco-2 permeability Mol. Inf. 32 459

31. Levatić J, Ćurak J, Kralj M, Šmuc T, Osmak M and Supek F 2013 Accurate models for P-gp drug recognition induced from a cancer cell line cytotoxicity screen J. Med. Chem. 565691

32. Lipinski C A, Lombardo F, Dominy B W and Feeney P J 2001 Experimental and computational approaches to estimate solubility and permeability in drug discovery and development settings Adv. Drug Deliv. Rev. 463
33. Hughes J D, Blagg J, Price D A, Bailey S, De Crescenzo G A, Devraj R V, Ellsworth E, Fobian Y M, Gibbs M E, Gilles R W, Greene N, Huang E, Krieger-Burke T, Loesel J, Wager T, Whiteley L and Zhang Y 2008 Physiochemical drug properties associated with in vivo toxicological outcomes Bioorg. Med. Chem. Lett. 184872

34. Pelzel H R, Schlamp C L and Nickells R W 2010 Histone $\mathrm{H} 4$ deacetylation plays a critical role in early gene silencing during neuronal apoptosis BMC Neurosci. 11 62

35. Edward H K and Li D 2002 Multivariate pharmaceutical profiling for drug discovery Curr. Top. Med. Chem. 287

36. Bradley C, Over B, Giordanetto F and Kihlberg J 2014 Oral druggable space beyond the rule of 5: Insights from drugs and clinical candidates Chem. Biol. 211115

37. Pham-The H, Garrigues T, Bermejo M, GonzálezÁlvarez I, Monteagudo M C and Cabrera-Pérez M Á 2013 Provisional classification and in silico study of biopharmaceutical system based on caco- 2 cell permeability and dose number Mol. Pharmaceutics 102445

38. Bohnert T and Gan L-S 2013 Plasma protein binding: From discovery to development J. Pharm. Sci. 102 2953

39. Smith D A, Beaumont K, Maurer T S and Di L 2015 Volume of distribution in drug design J. Med. Chem. 58 5691

40. Crivori P, Cruciani G, Carrupt P A and Testa B 2000 Predicting blood-brain barrier permeation from threedimensional molecular structure J. Med. Chem. 43 2204 